\title{
Globally Coupled Oscillator Networks
}

\author{
Eric Brown \\ Philip Holmes \\ Jeff Moehlis
}

To Larry Sirovich on the occasion of his 70th birthday

\begin{abstract}
We study a class of permutation-symmetric globally-coupled, phase oscillator networks on $N$-dimensional tori. We focus on the effects of symmetry and of the forms of the coupling functions, derived from underlying Hodgkin-Huxley type neuron models, on the existence, stability, and degeneracy of phase-locked solutions in which subgroups of oscillators share common phases. We also estimate domains of attraction for the completely synchronized state. Implications for stochastically forced networks and ones with random natural frequencies are discussed and illustrated numerically. We indicate an application to modeling the brain structure locus coeruleus: an organ involved in cognitive control.
\end{abstract}

\section{Introduction and background}

We consider networks of $N$ rotator oscillators with constant forcing and pairwise phase-difference and absolute-phase 'product' coupling, described by:

$$
\dot{\theta}_{i}=\omega_{i}+\frac{1}{N} \sum_{j=1}^{N} \alpha_{i j} f_{i j}\left(\theta_{j}-\theta_{i}\right)+h_{i}\left(\theta_{i}\right) \frac{1}{N-1} \sum_{j \neq i}^{N} \beta_{i j} g_{j}\left(\theta_{j}\right),
$$

where $\left(\theta_{1}, \ldots, \theta_{N}\right)^{\mathrm{T}} \in \mathrm{T}^{N}, \alpha_{i j}, \beta_{i j}$ and $f_{i j}, h_{i}, g_{j}$ are, respectively, coupling parameters and $2 \pi$-periodic functions, and $\omega_{i}$ are the natural frequencies of the uncoupled rotators. This paper focuses on networks with identical global (mean field) coupling, so that equation (1.1) becomes

$$
\dot{\theta}_{i}=\omega+\frac{\alpha}{N} \sum_{j=1}^{N} f\left(\theta_{j}-\theta_{i}\right)+h\left(\theta_{i}\right) \frac{\beta}{N-1} \sum_{j \neq i}^{N} g\left(\theta_{j}\right),
$$

although we include some results with randomly distributed frequencies $\omega_{i}$, and also with additive random noise. The denominators $(N, N-1)$ are introduced to normalize coupling effects. 
Rotator (phase-only) models of coupled oscillators have been widely studied, especially in the contexts of neuroscience and coupled Josephson junctions. The phase equations offer, respectively, significant simplification of more realistic neuron models of Hodgkin-Huxley or Fitzhugh-Nagumo type: see e.g. Murray [2001]; Keener and Sneyd [1998]; Hoppensteadt and Izhikevich [1997], and of the Josephson circuit equations: e.g. Watanabe and Strogatz [1994]; Watanabe and Swift [1997]; Weisenfeld, Colet, and Strogatz [1998]. In the case that the $N$ uncoupled oscillators have strongly attracting limit cycles in their full phase space, the persistence of normally hyperbolic invariant manifolds (Fenichel [1971]) under small perturbations (weak coupling) may be used to reduce the system to the $N$-torus by a suitable coordinate transformation. Two procedures for approximating the reduced system will be applied in Section 4 of this paper. The first is the 'strong attraction' (SA) method described in Ermentrout and Kopell [1990] and Hoppensteadt and Izhikevich [1997]; the second, a related 'phase response' (PR) technique, is given in e.g. Ermentrout [1996], Kuramoto [1997], and Kim and Lee [2000].

We were motivated to study systems of the form (1.1) by the study of Usher, Cohen, Servan-Schreiber, Rajkowsky, and Aston-Jones [1999], which presents data showing that neurons of the brain organ locus coeruleus (LC) in monkeys exhibit two distinct firing patterns corresponding to different behaviors evinced in cognitive tasks, cf. Grant, Aston-Jones, and Redmond [1988]; Aston-Jones, Rajkowski, and Alexinsky [1994]. These are designated as the phasic and tonic modes. In the latter, associated with labile behavior and poor task performance, $\mathrm{LC}$ neurons fire at a relatively high rate, but with little synchrony; in the former, associated with good performance, their average firing rate is lower but they display greater synchrony (i.e. higher correlation among individual firing times). See Figure 1.1(a). Moreover, firing patterns are more responsive to changes in stimulus in the phasic than in the tonic mode; see Figure 1.1(b,c). Thus, the LC has been proposed as a modulator involved in cognitive control, cf. Servan-Schreiber, Printz, and Cohen [1990].

The computational model constructed in Usher, Cohen, Servan-Schreiber, Rajkowsky, and Aston-Jones [1999] includes inhibitory synaptic and excitatory electrotonic coupling (Johnston and Wu [1997]), explicitly imposed refractory periods, and representations of rapid depolarization during action potentials to successfully reproduce these characteristics of the phasic and tonic modes; transitions between the two modes are effected by varying the degree of electrotonic coupling. However, the model's complexity makes analysis difficult, and we wish to develop a model that has similar behavior but is more amenable to mathematical study.

In this paper, we consider coupling functions motivated by two physically distinct mechanisms: (1) electrotonic or gap junction coupling, based on voltage differences between cells in electrical contact, and (2) spiketriggered synaptic transmission that releases a pulse of neurotransmitter 

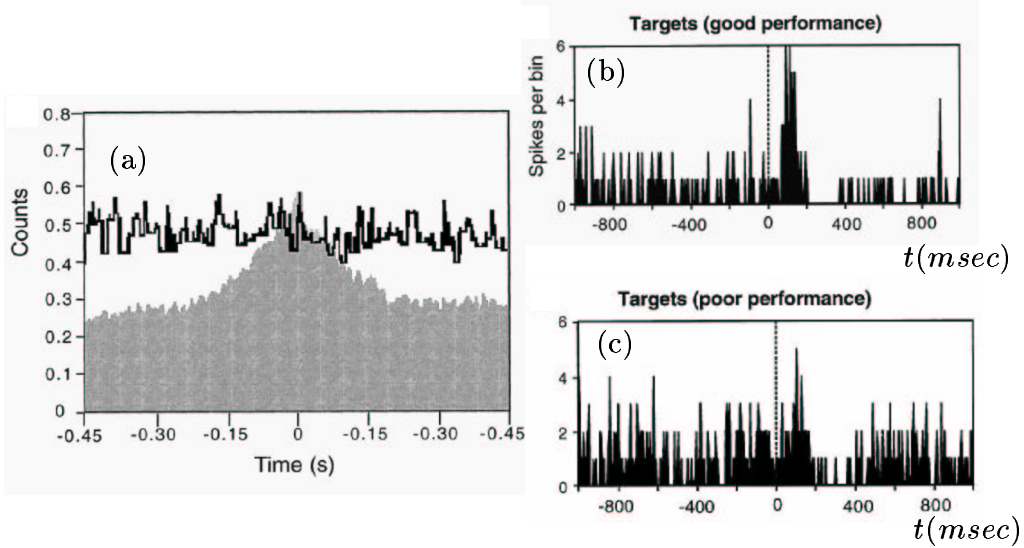

Figure 1.1. Reproduced from Usher, Cohen, Servan-Schreiber, Rajkowsky, and Aston-Jones [1999], with adapted caption. (a) Cross correlograms for two simultaneously recorded LC neurons during phasic LC mode (filled histogram) and tonic mode (line): central peak indicates increased synchrony in phasic mode. $(\mathrm{b}, \mathrm{c})$ Histograms of LC activity in phasic (b) and tonic (c) LC modes: psychological stimulus onset (which precedes the direct stimulus $I(t)$ considered in Section 4.4) is marked by dotted line, and enhanced response in phasic versus tonic mode is apparent. Average firing rate is lower in phasic mode.

across synaptic clefts. Electrotonic coupling is additive in the sense that the sum of voltage differences of all cells in contact with a given cell influence that cell; hence the first sum in equations (1.1) and (1.2). Synaptic coupling leads to absolute phase terms $\beta h_{i}\left(\theta_{i}\right) g_{j}\left(\theta_{j}\right)$ in (1.1-1.2). Intuitively, these arise because the primary effect on the post-synaptic cell occurs after the pre-synaptic cell fires, and therefore depends, via $g\left(\theta_{j}\right)$, on the latter's location on its phase circle. Coupling via a 'reversal potential' also depends upon the post-synaptic cell's phase through $h\left(\theta_{i}\right)$ (Ermentrout and Kopell [1990]; Taylor and Holmes [1998]); thus $h\left(\theta_{i}\right)$ multiplies the summed $g\left(\theta_{j}\right)$ 's, leading to the product coupling form of the second term.

We furthermore assume an additional separation of scales, taking electrotonic coupling to be weaker than synaptic, so that it can be averaged to give the phase-difference functions $\alpha f_{i j}$ without affecting $\beta h_{i} g_{j}$ at leading order; we are currently studying when the standard averaging theorems can be extended to make this rigorous. Sections 3 and 4 consider the dynamics of equation (1.1) for various values of $\alpha$ and $\beta$, without a priori restricting to the $|\alpha| \ll|\beta| \ll \mathcal{O}(1)$ required in this derivation of the phase equations.

When $\beta=0$ but frequencies differ between oscillators, equation (1.2) is referred to as the Kuramoto model (Kuramoto [1984]), on which there is an extensive literature; see the recent review of Strogatz [2000] and references therein (e.g. Crawford [1995]). Much of this work has been done in 
the continuum limit $N \rightarrow \infty$, and Strogatz [2000] adopts this viewpoint; specifically, stability analyses of some stationary (continuous) states are discussed. Finite-dimensional results, including a Liapunov function and dimension reduction, are found in the context of Josephson junction models in Watanabe and Strogatz [1994]. Many earlier studies take only the leading term in an odd Fourier expansion of $f$, so that $f(\cdot)=\sin (\cdot)$; as we shall see this is a very degenerate case for the mean field coupled system (1.2) (e.g. Nichols and Wiesenfeld [1992]; Golomb, Hansel, Shraiman, and Sompolinsky [1992]). Moreover, as shown in Izhikevich [2000], relaxation oscillators of Hodgkin-Huxley or Fitzhugh-Nagumo type lead to much richer phase difference functions than $\sin (\cdot)$. Others have recognized the importance of higher Fourier harmonics: see Daido [1994]; Golomb, Hansel, Shraiman, and Sompolinsky [1992]; Nichols and Wiesenfeld [1992]; Watanabe and Swift [1997]. Additional work on finite dimensional oscillator networks includes Kopell and Ermentrout [1990]; Kopell, Ermentrout, and Williams [1991]; Kopell and Ermentrout [1994], which consider directed coupling, Bressloff and Coombes [1998], which considers integrate-and-fire models derived from coupled spiking neurons, and Okuda [1993], which will be discussed in Section 2. Shortly before this paper was submitted, we learned of recent work of Chow and Kopell [2000], in which the effects of spike shape on electrotonically coupled integrate-and-fire networks are studied. They find that the existence and stability of splay states depends on the spike shape in a manner that would be interesting to compare with the present results.

The present paper draws on Ashwin and Swift [1992], which addresses a class of $S_{N} \times T^{1}$-equivariant oscillator networks (of which (1.2) is an example when $\beta=0$ ). We now summarize the properties of symmetric dynamical systems necessary to present and apply these results; for more background, see Golubitsky, Stewart, and Schaeffer [1988].

Consider the ODE

$$
\frac{d x}{d t}=f(x), x \in \text { manifold } M,
$$

and let $\Gamma$ be a group acting on $M$. The ODE is said to be $\Gamma$-equivariant if $f$ commutes with the group action, i.e.

$$
f(\gamma x)=\widehat{\gamma} f(x) \forall \gamma \in \Gamma, x \in M,
$$

where the derivative map $\widehat{\gamma}$ (Arnold [1973]) acts on the tangent space TM; for linear actions of $\gamma, \widehat{\gamma}=\gamma$. The symmetry of a solution $x_{0} \in M$ is characterized by the isotropy subgroup $\Sigma_{x_{0}}=\left\{\gamma \in \Gamma: \gamma x_{0}=x_{0}\right\}$, that is, the set of all group elements which leave the solution $x_{0}$ unchanged. Associated with an isotropy subgroup is a fixed point subspace Fix $\left[\Sigma_{x_{0}}\right]=$ $\left\{x \in M: \sigma x=x \quad \forall \sigma \in \Sigma_{x_{0}}\right\}$ : the set of points fixed by all elements of $\Sigma_{x_{0}}$. Two immediate consequences of $\Gamma$-equivariance are that (1) for any 
solution $x(t)$ to equation (1.3), $\gamma x(t)$ is also a solution, and (2) fixed-point subspaces are invariant under the flow generated by $f$. We will refer to this latter property as dynamical invariance. As in Ashwin and Swift [1992], we study special classes of symmetric systems defined by the following groups: the circle group $T^{1}=\{\delta: \delta \in[0,2 \pi)\}$ (with action on $\mathrm{T}^{N}, \theta_{i} \mapsto \theta_{i}+\delta, \forall i$ ), the cyclic subgroups $Z_{m} \in T^{1}$ (with action $\theta_{i} \mapsto \theta_{i}+2 \pi / m$ ), and the subgroups of permutations on $j$-many coordinates, $S_{j}$.

The remainder of the paper proceeds as follows. In Section 2 we study (1.2) with $\beta=0\left(S_{N} \times T^{1}\right.$ equivariant $)$, emphasizing the influence of general coupling functions and obtaining additional results for odd functions $f$. In succeeding sections the symmetries are gradually relaxed. In Section 3 this is done by breaking $T^{1}$ equivariance through re-introduction of $h\left(\theta_{i}\right) g\left(\theta_{j}\right)$ terms. In Section 4 we break $S_{N}$ equivariance by introducing a random distribution of frequencies as well as random excitation, and apply our model to the LC. Thus, Sections 2 and 3 are largely abstract and general, while Section 4 concerns specific 'neural' coupling. Conclusions are drawn and future directions noted in Section 5. Our major contributions to this survey on globally coupled oscillators include the implications of gradient dynamics for the existence of families of equilibria, nonlinear stability results for the synchronized state, and the analysis of a two-parameter $(\alpha, \beta)$ system, including the influence of noise, in relation to the LC model.

In a series of recent papers (e.g. Omurtag, Knight, and Sirovich [2000b]; Sirovich, Knight, and Omurtag [2000]; Omurtag, Kaplan, Knight, and Sirovich [2000a]), Larry Sirovich and his colleagues have also addressed problems in neurobiology; specifically, modeling networks of neurons in visual cortex. They employ integrate-and-fire units, and develop an evolution equation for the probability $\rho(V, t) d V$ of finding a neuron with membrane potential $V \in[V, V+d V]$ at time $t$. We hope that our study, which takes a different, and, we believe, a complementary viewpoint, will at once serve as a suitable tribute, a stimulus for future work, and a fond reminder of turbulent interactions past.

\section{$2 \quad S_{N} \times T^{1}$ phase difference oscillator systems}

This section treats the system of $N$ oscillators,

$$
\dot{\theta}_{i}=\omega+\frac{\alpha}{N} \sum_{j=1}^{N} f\left(\theta_{j}-\theta_{i}\right), \quad i=1, \ldots, N,
$$

where $f(\cdot)$ is assumed to be continuously differentiable and $2 \pi$-periodic. Transforming to coordinates $\phi_{i}=\theta_{i}-\omega t$ rotating with the common natural 
frequency, equation (2.1) becomes

$$
\dot{\phi}_{i}=\frac{\alpha}{N} \sum_{j=1}^{N} f\left(\phi_{j}-\phi_{i}\right)
$$

Denoting the phase differences $\phi_{j}-\phi_{i} \equiv \phi_{j i}$, we seek 'diagonal flow' periodic solutions $\bar{\phi}$ of equation (2.2) in the form

$$
\dot{\bar{\phi}}_{i}=\frac{1}{N} \sum_{j=1}^{N} f\left(\bar{\phi}_{j i}\right)=c, \quad i=1, \ldots, N
$$

where $c$ is a constant, nonzero in general. These solutions are also periodic for (2.1), and, employing a second rotating frame $\theta_{i}-(\omega+c) t$, they become fixed points. Since the derivatives $f^{\prime}\left(\phi_{j i}\right)$ are time-independent, eigenvalue calculations suffice to determine the stability of such solutions. Equation (2.3) determines the $N-1$ phase differences, without loss of generality leaving the phase $\phi_{1}$ unspecified, as expected from the $T^{1}$ symmetry of equation (2.1).

We began this work by determining the existence and stability of diagonal flow solutions to equation. (2.2). While we later found many of the following results in the literature, no unified presentation appears to exist, so we provide a summary here, including extensions and new examples of our own. Proofs are only sketched.

\subsection{Gradient property for odd phase-difference coupling}

If $f(\cdot)$ is odd, we observe as in Theorem 9.15 of Hoppensteadt and Izhikevich [1997] that:

2.1 Proposition. Equation (2.2) is a gradient dynamical system on $\mathrm{T}^{N}$ with potential

$$
V=\frac{\alpha}{N} \sum_{i=1}^{N-1} \sum_{j=i+1}^{N} F\left(\phi_{j}-\phi_{i}\right), \text { where } f(\theta)=F^{\prime}(\theta)
$$

Proof. Note that

$$
-\frac{\partial V}{\partial \phi_{i}}=\frac{\alpha}{N} \sum_{j<i} f\left(\phi_{j}-\phi_{i}\right)-\frac{\alpha}{N} \sum_{j>i} f\left(\phi_{i}-\phi_{j}\right)
$$

the oddness of $f$ implies that $\dot{\phi}_{i}=-\partial V / \partial \phi_{i}$. 
Proposition 2.1 implies that

$$
\dot{V}=\sum_{i=1}^{N} \frac{\partial V}{\partial \phi_{i}} \dot{\phi}_{i}=-\sum_{i=1}^{N} \dot{\phi}_{i}^{2} \leq 0,
$$

with equality only at equilibria. Thus, equation $(2.2)$ with odd $f(\cdot)$ has no periodic or homoclinic orbits or heteroclinic cycles: all solutions approach equilibria, and almost all approach stable equilibria. In particular,

2.2 Corollary. For $f$ odd, equation (2.3) has no solutions unless $c=0$.

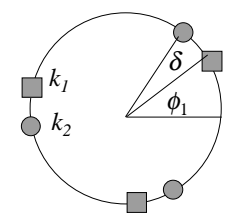

a)

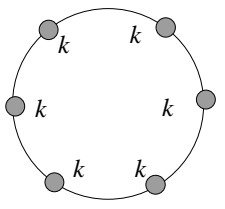

b)

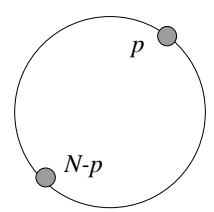

c)

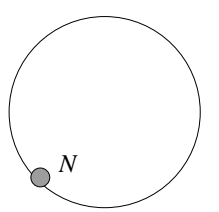

d)

FIGURE 2.1. Illustrations of equilibria fixed under the actions of various isotropy subgroups. (a) An element of $\operatorname{Fix}\left[\left(S_{k_{1}} \times S_{k_{2}}\right)^{3} \rtimes Z_{3}\right]$. Each square represents $k_{1}$ oscillators mutually in phase, and successive squares denote groups differing in phase by $2 \pi / 3$ (similar for circles). Elements of this fixed point subspace are parameterized by two angles $\phi_{i}$ and $\delta$, so it is a 2-torus. (b) $\left(S_{k}\right)^{m} \rtimes Z_{m}$ (rotating block modes) with $m=6$, (c) $S_{p} \times S_{N-p}$ (two-block modes), and (d) $S_{N}$ (in-phase mode).

We begin by stating two results from Ashwin and Swift [1992] which hold for arbitrary $2 \pi$-periodic, continuously differentiable coupling functions $f(\cdot)$ :

2.3 Theorem. (Ashwin and Swift [1992]) Every isotropy subgroup of a general $S_{N} \times T^{1}$-equivariant vector field is of the form:

$$
\Sigma_{\mathbf{k}, m} \equiv\left(S_{k_{1}} \times \cdots \times S_{k_{l_{B}}}\right)^{m} \rtimes Z_{m},
$$

where $N=m\left(k_{1}+\cdots+k_{l_{B}}\right)$, and $\rtimes$ denotes the semi-direct product.

The fixed-point subspace $\operatorname{Fix}\left[\Sigma_{\mathbf{k}, m}\right]$ may be thought of as being partitioned into $m$ blocks each containing $k=\left(k_{1}+\cdots+k_{l_{B}}\right)$ oscillators. The solution is invariant under time shifts of the period divided by $m$, coupled with a cyclic permutation of the blocks, giving the $Z_{m}$ symmetry. Each block is partitioned into clusters of $k_{i}$ oscillators, and the solution is invariant under permutations of oscillators within these clusters, giving the $S_{k_{1}} \times \cdots \times S_{k_{l_{B}}}$ 
symmetry. These permutations all commute, hence the direct products, while the $Z_{m}$ symmetry does not commute with the permutations, hence the semi-direct product. Overall, the fixed-point subspace $\operatorname{Fix}\left[\Sigma_{\mathbf{k}, m}\right]$ is an $l_{B}$-torus: there are $l_{B}-1$ degrees of freedom setting the spacings between the blocks, plus an additional degree of freedom determining a 'reference' $\phi_{1}$ (see Figure 2.1(a) for an example); this represents the $T^{1}$ group orbit and will be associated with a unit Floquet multiplier.

2.4 Theorem. (Ashwin and Swift [1992]) Every $\operatorname{Fix}\left[\left(S_{k}\right)^{m} \rtimes Z_{m}\right]$ with $m k=N$ and $\operatorname{Fix}\left[\left(S_{k_{1}} \times S_{k_{2}}\right)^{m} \rtimes Z_{m}\right]$ with $m\left(k_{1}+k_{2}\right)=N$, generically contains a periodic orbit with diagonal flow.

Ashwin and Swift [1992] prove this theorem by noting that, without loss of generality, the phases of the oscillators can be ordered as $\phi_{1} \leq \phi_{2} \leq$ $\cdots \leq \phi_{N} \leq \phi_{1}+2 \pi$. The oscillators retain their ordering under the dynamics, i.e., they can never 'pass' each other, since this would involve crossing an invariant fixed-point subspace. Projecting the phases onto the manifold $\phi_{1}=0$ (by subtracting the instantaneous value of $\phi_{1}$ from each phase) gives a simplex called the 'canonical invariant region' (CIR). The intersection of $\operatorname{Fix}\left[\left(S_{k}\right)^{m} \rtimes Z_{m}\right]$ with the CIR is a zero-dimensional invariant subspace, i.e., an equilibrium. In the unprojected system, this corresponds to a periodic orbit (or a circle of equilibria if $\phi_{1}=0$ for all time) with isotropy $\left(S_{k}\right)^{m} \rtimes Z_{m}$. Furthermore, the intersection of Fix $\left[\left(S_{k_{1}} \times S_{k_{2}}\right)^{m} \rtimes Z_{m}\right]$ with the CIR is a one-dimensional line segment. The end points of this segment have isotropy $\left(S_{k_{1}+k_{2}}\right)^{m} \rtimes Z_{m}$, and are equilibria with stability in the direction of the line segment determined by the same eigenvalue. Provided this eigenvalue does not vanish (this is the nondegeneracy condition satisfied for generic functions $f$ ), this can only happen if there is at least one equilibrium in the interior of the line segment. In the original system, this corresponds to a periodic orbit (or one-torus of equilibria if $\dot{\phi}_{1}=0$ for all time) with isotropy $\left(S_{k_{1}} \times S_{k_{2}}\right)^{m} \rtimes Z_{m}$. If $k_{1}=k_{2}$, the midpoint of the line segment is an equilibrium with isotropy $\left(S_{k_{1}}\right)^{2 m} \rtimes Z_{2 m}$; this can serve as the necessary equilibrium in the interior of the line segment.

Ashwin and Swift [1992] developed their proof for systems coupled with general $T^{1}$-equivariant functions. The special additive, pairwise-coupled form of the coupling in (2.1) allows a much simpler argument to prove (a restricted version of) Theorem 2.4. For a $\left(S_{k_{1}} \times S_{k_{2}}\right)^{m} \rtimes Z_{m}$ solution with clusters separated by phase $\delta$ (see Figure 2.1(a)) to have diagonal flow requires $\dot{\phi}_{i} \equiv c(\delta) \forall i$, for some fixed $\delta$. This condition reduces to

$$
c_{1}(\delta)=c_{2}(\delta), \text { where }
$$




$$
\begin{aligned}
& c_{1}(\delta)=k_{1} \sum_{j=0}^{m-1} f\left(\frac{2 \pi j}{m}\right)+k_{2} \sum_{j=0}^{m-1} f\left(\frac{2 \pi j}{m}+\delta\right) \\
& c_{2}(\delta)=k_{2} \sum_{j=0}^{m-1} f\left(\frac{2 \pi j}{m}\right)+k_{1} \sum_{j=0}^{m-1} f\left(\frac{2 \pi j}{m}-\delta\right)
\end{aligned}
$$

are the (constant) phase velocities for oscillators in $k_{1}$ or $k_{2}$-clusters, respectively (cf. Kim and Lee [2000] for $m=1$ ). A quick sketch shows that at least one $\delta \in(0,2 \pi / m)$ satisfying $(2.7)$ must exist if $c_{1}^{\prime}(0) / k_{2}=-c_{2}^{\prime}(0) / k_{1}$ is nonzero, since $c_{1,2}^{\prime}(0)=c_{1,2}^{\prime}(2 \pi / m)$. Thus, the nondegeneracy condition becomes $\sum_{j=0}^{m-1} f^{\prime}\left(\frac{2 \pi j}{m}\right) \neq 0$; for $S_{N-p} \times S_{p}$ solutions, $m=1$, implying $f^{\prime}(0) \neq 0$. Further, for $\left(S_{k}\right)^{m} \rtimes Z_{m}$ rotating blocks the equality $\dot{\phi}_{i} \equiv \gamma$ is automatic. Finally, we note that if $k_{1}=k_{2}, \delta=\pi / m$ always satisfies (2.7), so that the corresponding $\left(S_{k_{1}} \times S_{k_{1}}\right)^{m} \rtimes Z_{m}$ solutions may also have symmetry $\left(S_{k_{1}}\right)^{2 m} \rtimes Z_{2 m}$.

These arguments extend in a natural way to show the existence of weak solutions to the partial differential equations derived from (2.1) as $N \rightarrow \infty$ (Crawford and Davies [1999]). These are symmetrically-spaced combinations of delta distributions rotating at the frequency $c(\delta)$ found above, with the $k_{j}$-cluster distributions weighted by $k_{j}(N) / m\left(k_{1}(N)+k_{2}(N)\right), j=1,2$. Here, $k_{j}(N)$ is the number of oscillators in a cluster when the total number of oscillators is $N$, and the $N \rightarrow \infty$ limit is taken over a subsequence of configurations (E [2001]) with constant $k_{j}(N) / m\left(k_{1}(N)+k_{2}(N)\right)$ such that $m$ (fixed) divides $k_{1}(N)+k_{2}(N)$. Under the same nondegeneracy conditions as above, their existence may be shown for any values of $k_{j} / m\left(k_{1}+k_{2}\right)$ and any $m$. Furthermore, if $f$ lacks $m$-th Fourier harmonics and their multiples, families of $Z_{m}$-symmetric solutions analogous to the fixed tori of Section 2.4 also exist. A study of the stability of these solutions, their persistence under the introduction of a (diffusive) noise term, and associated convergence issues as $N \rightarrow \infty$ is in progress.

For the special case of odd phase-difference coupling, Proposition 2.1 ensures the existence of additional equilibria:

2.5 Theorem. For the phase-difference system (2.2) with $f$ odd, every $\operatorname{Fix}\left[\left(S_{k_{1}} \times \cdots \times S_{k_{l_{B}}}\right)^{m} \rtimes Z_{m}\right]$ with $m\left(k_{1}+\cdots+k_{l_{B}}\right)=N$ contains at least one equilibrium.

Proof. Let $\widehat{V}_{\mathbf{k}, m}$ denote the restriction of $V$ from $(2.4)$ to the $l_{B}$-torus $\Sigma_{\mathbf{k}, m} \cdot \widehat{V}_{\mathbf{k}, m}$ is a continuous function on the (flow-invariant) compact set $\Sigma_{\mathbf{k}, m}$ and thus possesses a minimum $\bar{\phi}$ on $\Sigma_{\mathbf{k}, m}$. Consider a trajectory $\phi(t) \in \Sigma_{\mathbf{k}, m}$ starting at $\bar{\phi}$ at $t=0$. Since $\dot{V}=-\sum_{i=1}^{N} \dot{\phi}_{i}{ }^{2}$, if $\phi(t) \neq \bar{\phi}$ then $V(\phi(t))<V(\bar{\phi}), \forall t>0$. This contradicts the assumption that $\bar{\phi}$ is a minimum for $\mathrm{V}$, so $\bar{\phi} \in \Sigma_{\mathbf{k}, m}$ must be a fixed point for equation (2.2).

Associated with any of the equilibria above, we expect at least one zero 
eigenvalue and a circle of equilibria corresponding to its $T^{1}$ group orbit.

Despite the variety of these equilibria, we can prove a result on the nonexistence of fixed points in a region surrounding the in-phase solution. Define the open $N$-cylinder $\mathcal{C}_{R_{1}} \triangleq\left\{\theta \mid d\left(\theta, \theta_{d}(\psi)\right) \leq R_{1}\right.$ for some $\left.\psi \in[0,2 \pi]\right\}$. Here, $d(\cdot, \cdot)$ is the Euclidian metric on $\mathcal{R}^{N}$ (and hence on $\mathrm{T}^{N}$ ) and $\theta_{d}(\psi)$ is the $N$-vector with all coordinates equal to $\psi$ (so that the axis of $\mathcal{C}_{R_{1}}$ is the diagonal $\mathcal{D} \triangleq\left\{\theta \mid \theta_{i}=\theta_{j} \forall i, j\right\}$; see Figure 2.2).

2.6 Proposition. Let $R_{1}>0$ be such that either $f$ restricted to $\left(0,2 R_{1}\right)$ or $f$ restricted to $\left(-2 R_{1}, 0\right)$ is of one sign (i.e., $f$ is strictly negative or positive in the region). Then there are no fixed points in $\mathcal{C}_{R_{1}} \backslash \mathcal{D}$.

Proof. First we note that for $\phi \in \mathcal{C}_{R_{1}}$, there exists a $\psi$ s.t. $\phi \in B_{R_{1}}(\psi) \triangleq$ $\left.\left\{\phi \mid d\left(\phi, \theta_{d}(\psi)\right)<R_{1}\right)\right\}$. Thus in particular $\left|\phi_{j}-\psi\right|<R_{1} \forall j$; summing two of these inequalities and applying the triangle inequality gives $\left|\phi_{j i}\right|<$ $2 R_{1} \forall i, j$. Next, consider an arbitrary $N$-vector $\phi \in \mathcal{C}_{R_{1}} \backslash \mathcal{D}$, where without loss of generality $\phi_{1} \leq \phi_{2} \leq \cdots \leq \phi_{N}$. If the chosen interval in the hypothesis is $\left(0,2 R_{1}\right)$, note that $2 R_{1}>\phi_{j}-\phi_{1} \geq 0 \forall j$ and $\phi_{j}-\phi_{1}>0$ for at least one $j$ (since $\phi \notin \mathcal{D}$ ). Thus, $\dot{\phi}_{1}=\frac{\alpha}{N} \sum_{j=1}^{N} f\left(\phi_{j}-\phi_{1}\right) \neq 0$, because each term in the sum is either zero or of the same sign (the continuity of $f$ implies that $f(0)$ is either 0 or of the same sign as $f(\phi)$ for $\left.\phi \in\left(0,2 R_{1}\right)\right)$ and at least one term is nonzero. If the chosen interval is $\left(-2 R_{1}, 0\right)$, we use the facts that $0 \geq \phi_{j}-\phi_{N}>-2 R_{1} \forall j$ and $\phi_{j}-\phi_{N}<0$ for at least one $j$. Similarly, then, $\dot{\phi_{N}}=\frac{\alpha}{N} \sum_{i=1}^{N} f\left(\phi_{j}-\phi_{N}\right) \neq 0$. Hence $\phi$ is not a fixed point.

This result does not exclude orbits with nonzero diagonal flow, which may exist within $\mathcal{C}_{R_{1}} \backslash \mathcal{D}$ if $f$ is not odd.

We now consider the stability of some of the solutions found above.

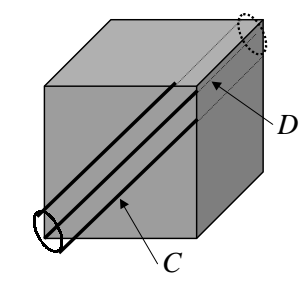

Figure 2.2. The diagonal $\mathcal{D}$ and cylinder $\mathcal{C}$ of Proposition 2.6. The cube represents the $N$-torus. 


\subsection{Stability of periodic orbits}

\section{Rotating blocks}

We study solutions with isotropy $\left(S_{k}\right)^{m} \rtimes Z_{m}$ (rotating block modes), $S_{p} \times$ $S_{N-p}$ (two-block modes), and $S_{N}$ (in-phase mode). Since the Jacobian is constant along these periodic orbits with diagonal flow, this problem reduces to computation of eigenvalues (Ashwin and Swift [1992]). Stability will be discussed in terms of orbital stability, which implies asymptotic stability with respect to all perturbations transverse to the (continuous) $T^{1}$ group orbit of the solution (hence excluding the corresponding zero eigenvalue). Note that if $c \neq 0$ in equation (2.3), the group orbit and periodic orbit coincide.

To state some of the stability results, it is useful to express the coupling function in a Fourier series with coefficients $b_{l}^{o}$ and $b_{l}^{e}$ :

$$
f\left(\phi_{j i}\right)=\sum_{l=0}^{\infty}\left(b_{l}^{o} \sin \left(l \phi_{j i}\right)+b_{l}^{e} \cos \left(l \phi_{j i}\right)\right) .
$$

For $\left(S_{k}\right)^{m} \rtimes Z_{m}$ symmetric solutions we have, as in Okuda [1993] and (for $m=1$ ) Watanabe and Swift [1997]:

2.7 Proposition. Let $N=m k$ and let $\bar{\phi}$ be an $\left(S_{k}\right)^{m} \rtimes Z_{m}$-invariant fixed point or periodic orbit with diagonal flow. Then the eigenvalues of the Jacobian $J(\bar{\phi})$ obtained by linearization of Equation (2.2) are

$$
\begin{aligned}
& \lambda=\lambda_{0}=0, \quad \text { with multiplicity } 1 \\
& \lambda=\lambda_{r}^{j}, \quad j=1, \ldots, m-1 \text { : 'rotation eigenvalues' } \\
& \left.\lambda=\lambda_{p}, \quad \text { with multiplicity } m(k-1) \text { : 'permutation eigenvalues' }\right\} \\
& \lambda_{r}^{j}=\frac{\alpha}{m} \sum_{k=1}^{m-1} f^{\prime}\left(\frac{2 \pi k}{m}\right)\left(\exp \left(\frac{2 \pi k j i}{m}\right)-1\right) \\
& =\frac{\alpha}{2}\left(\sum_{l \in \mathcal{M}(m)_{1}^{j}} l\left(b_{l}^{o}+i b_{l}^{e}\right)+\sum_{l \in \mathcal{M}(m)_{2}^{j}} l\left(b_{l}^{o}-i b_{l}^{e}\right)-2 \sum_{l \in \mathcal{M}(m)} l b_{l}^{o}\right) \\
& \lambda_{p}=-\frac{\alpha}{m} \sum_{k=0}^{m-1} f^{\prime}\left(\frac{2 \pi k}{m}\right)=-\alpha \sum_{l \in \mathcal{M}(m)} b_{l}^{o} l \\
& \mathcal{M}(m)_{1}^{j}=\{m h-j \mid h=1,2, \ldots\}, \quad \mathcal{M}(m)_{2}^{j}=\{m h+j \mid h=0,1,2, \ldots\}, \\
& \mathcal{M}(m)=\{m h \mid h=1,2, \ldots\} \text {. }
\end{aligned}
$$


The 'rotation' and 'permutation' terminology is due to Ashwin and Swift [1992], where general formulae for eigenvalues and eigenvectors are presented. The proof of Proposition 2.7 repeatedly uses the following simple fact. Define the set $\mathcal{M}=\{l \mid l=q m$ for some $q \in \mathbb{Z}\}$ and let $\gamma=\exp (2 \pi i / m)$; then for $\bar{l} \in \mathbb{Z} \backslash \mathcal{M}, \sum_{r=1}^{m-1} \gamma^{\bar{l} r}=-1$. From here, the $m \times m$-blocked structure of the Jacobian along with results on the eigenvectors of Toeplitz matrices leads to the desired conclusion.

If $m=1$, then the proposition addresses the $S_{N}$-invariant (in-phase) solutions. Here there are no rotation eigenvalues, and the permutation eigenvalues are simply

$$
\lambda_{p}=-\alpha f^{\prime}(0),
$$

with multiplicity $N-1$. Nonlinear stability of these solutions is discussed in Section 3.2 (Proposition 3.2 with $\beta=0$ ). At the other extreme, if $m=N$, the proposition addresses the $Z_{N}$-invariant solutions in which the phases of the oscillators are equally spaced; these are called 'rotating wave' solutions by Ashwin and Swift [1992], and correspond to the 'splay state' in the Josephson junction literature. In this case, there are no permutation eigenvalues, and (2.11) reduces to equation (63) of Watanabe and Swift [1997].

We now give examples to illustrate several interesting stability behaviours implied by Proposition 2.7. Including only the first harmonic in the coupling function $\left(f(\cdot)=b_{1}^{o} \sin (\cdot)+b_{1}^{e} \cos (\cdot)\right)$, we have for $m>1$ :

$$
\begin{aligned}
\lambda_{p} & =0, \text { with multiplicity } m(k-1) \\
\lambda_{r}^{j} & = \begin{cases}\frac{\alpha}{2}\left(b_{1}^{o}-i b_{1}^{e}\right) \text { and } \frac{\alpha}{2}\left(b_{1}^{o}+i b_{1}^{e}\right) & \text { for } j=1 \text { and } m-1 \\
0 & \text { otherwise (multiplicity } m-3),\end{cases}
\end{aligned}
$$

in addition to $\lambda_{0}$. In this case the $\left(S_{k}\right)^{m} \rtimes Z_{m}$ solutions are highly degenerate and, for $\alpha b_{1}^{o}>0$, unstable. For $m=N(k=0)$, there are $N-2$ zero eigenvalues. This result is well-known from the Josephson junction literature; in Watanabe and Strogatz [1994], it is shown to be related to the integrability of the equations for this choice of $f$.

On the other hand, we note that inclusion of higher harmonics in $f(\cdot)$ generically unfolds the degeneracy in the sense that all but one $\left(\lambda_{0}\right)$ of the eigenvalues become nonzero, implying instability or orbital stability. For example, adding the $m$ th harmonic $\left(f(\cdot)=b_{1}^{o} \sin (\cdot)+b_{1}^{e} \cos (\cdot)+b_{m}^{o} \sin (m \cdot)+\right.$ $\left.b_{m}^{e} \cos (m \cdot) ; m \neq 1\right)$, we obtain

$$
\begin{aligned}
\lambda_{p} & =-\alpha b_{m}^{o} m, \text { with multiplicity } m(k-1) \\
\lambda_{r}^{j} & = \begin{cases}\alpha\left[\frac{1}{2}\left(b_{1}^{o}-i b_{1}^{e}\right)-b_{m}^{o} m\right], c . c . & \text { if } j=1, m-1 \\
-\alpha b_{m}^{o} m & \text { otherwise (multiplicity } m-3),\end{cases}
\end{aligned}
$$

so that any $\left(S_{k}\right)^{m} \rtimes Z_{m}$ solution is orbitally stable if $\alpha b_{m}^{o}>\alpha \frac{b_{1}^{o}}{2 m}$ and $\alpha b_{m}^{o}>0$. We also note that for coupling functions whose harmonic indices 
belong entirely to $\mathcal{M}(l)$, any oscillator may be individually translated from a $\left(S_{k}\right)^{m} \rtimes Z_{m}$ solution by a multiple of $\frac{2 \pi}{l}$ to give another equilibrium. These translations give a total of $l^{N}$ fixed points, each with identical stability (due to the $\frac{2 \pi}{l}$ periodicity of $f$ ).

The calculations proving Proposition 2.7 show which eigenvectors correspond to zero eigenvalues and hence along which directions there may be continuous families of equilibria. For example, with $k=1, m=N=4$ and $f(\cdot)=\sin (\cdot)$, the nondiagonal zero eigenvector is $(1,-1,1,-1)^{\mathrm{T}}$, which reflects the fact that equilibrium is preserved if 'diametrically-opposite' pairs of oscillators are rotated independently.

\section{Two-block periodic orbits}

For $f^{\prime}(0) \neq 0$, equation (2.13) guarantees that the $S_{N}$-invariant solutions satisfy the nondegeneracy assumption of Theorem 2.4. Then, the Theorem (with $m=1$ ) implies that for some $\delta(p)>0$, equation (2.2) has periodic orbits with $\phi_{j i} \in\{0, \delta(p), 2 \pi-\delta(p)\}$ for all $i, j$. This occurs when two blocks of $p$ and $N-p$ identical-phase oscillators are mutually out of phase by $\delta$; to avoid redundancy, we restrict $0 \leq p \leq\lfloor N / 2\rfloor$. The Jacobian from linearizing around a $S_{N-p} \times S_{p}$ solution has a four-blocked structure which yields:

2.8 Proposition. (Kim and Lee [2000]) Let $\bar{\phi}$ be an $\left(S_{p} \times S_{N-p}\right)$-invariant solution and $0 \leq p \leq\lfloor N / 2\rfloor$. Then for $p \geq 1$ the eigenvalues of the Jacobian from equation (2.2) are:

$$
\begin{array}{lll}
\lambda_{1}=\alpha\left(b-\frac{p}{N}(a+b)\right), & \text { with multiplicity } & p-1 \\
\lambda_{2}=\alpha\left(\frac{p}{N}(a+c)-a\right), & \text { with multiplicity } & N-p-1 \\
\lambda_{3}=0, & \text { with multiplicity } 1 \\
\lambda_{4}=\alpha\left(\frac{N-p}{N} b+\frac{p}{N} c\right), & & \text { with multiplicity } 1
\end{array}
$$

Here, $a=f^{\prime}(0), b=-f^{\prime}(\delta(p)), c=-f^{\prime}(-\delta(p))$.

If $f(\cdot)$ is odd, two-block states with $\delta=\pi$ exist for any $p$ since $f(0)=$ $f(\pi)=0$; we write $\delta \neq \delta(p)$ to indicate this $p$-independence of $\delta$. Oddness of $f$ also implies $b=c$. This case was studied in Okuda [1993], where expressions corresponding to (2.14) are presented.

2.9 Corollary. Assume that $b=c, \delta \neq \delta(p)$, and that $a, b>0$. If $\alpha>0$, the two-block equilibria of equation (2.2) are orbitally stable if and only if $p=0$. If $\alpha<0$, the equilibria are stable if and only if $p \neq 0$ and $a<b p /(N-p)$, if the equilibria are stable for $p=k$ for some $k \leq\lfloor N / 2\rfloor$, then they are stable for $p>k$.

Proof. The results for $\alpha>0$ are immediate from $\lambda_{4}$ of equation (2.14) and (2.13). For $\alpha<0$, we note that $\lambda_{1,2} \leq 0$ implies $N a \leq p(a+b) \leq N b$. Upon rearranging, this yields $a \leq b(N-p) / p$ and $a \leq b p /(N-p)$; for $p$ in the given range, the latter inequality implies the first, and for fixed $a$ 


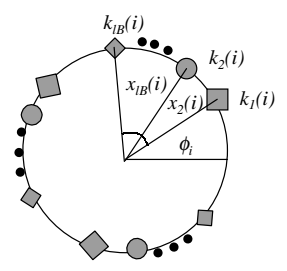

Figure 2.3. The labeling scheme used in Proposition 2.10. Given the reference index $i$ corresponding to the $\dot{\phi}_{i}$ being computed, blocks of oscillators are numbered by the index $q$ in a counterclockwise fashion, starting with $q=1$ for the block containing $\phi_{i}$ itself. Each block contains $k_{q}(i)$ oscillators and is separated from its neighbor by the angle $x_{q}(i)$ (by definition $x_{1}(i) \equiv 0$ ).

and $b$ it is clear that if the second inequality is satisfied for $p=k$, then it continues to be satisfied as $p$ increases. In this case $\lambda_{1}, \lambda_{2}$ and $\lambda_{4}$ are all strictly negative, leading to the Corollary.

We remark that if $a, b<0$, the sign of $\alpha$ may be switched and the Corollary applied, and that the result that stability of equilibria for $p=k$ implies stability for $p=N / 2$ is stated in Okuda [1993].

The corollary indicates that for $\alpha<0$ and under certain conditions on $a, b$, and $N$, orbital stability of two-block fixed points can change as $p$ is varied. For example, if $a=1, b=2$, and $N=5$, the equilibria are unstable for $p=0,1$ but are stable for $p=2$. In the special case $a=b=c$ (which occurs, for example, if $f(\cdot)=\sin (\cdot))$, note that $\lambda_{1}=-\lambda_{2}=\alpha(a-2 p / N)$, $\lambda_{4}=\alpha a$; thus the fixed points are unstable unless $\alpha a<0, N$ is even and $p=N / 2$, in which case they are neutrally stable with $N-1$ zero eigenvalues. As above, inclusion of higher harmonics in the Fourier series for $f(\cdot)$ generically unfolds this degeneracy.

We close this subsection by remarking that techniques used to prove Propositions 2.7 and 2.8 could in principle be extended to calculate the stability of general $\left(S_{k_{1}} \times S_{k_{2}}\right)^{m} \rtimes Z_{m}$ solutions for $m>1$, where $m\left(k_{1}+\right.$ $\left.k_{2}\right)=N$. We refer the reader to Ashwin and Swift [1992] for the specific example $\left(S_{2} \times S_{1}\right)^{3} \rtimes Z_{3}$.

\subsection{Existence of fixed $l_{B}$-tori}

2.10 Proposition. For $\phi$ contained in an invariant $l_{B}$-torus $\operatorname{Fix}\left[\left(S_{k_{1}} \times\right.\right.$ $\left.\cdots \times S_{k_{l_{B}}}\right)^{m} \rtimes Z_{m}$ ] with $N=m\left(k_{1}+\cdots+k_{l_{B}}\right)$, equation (2.2) reduces to:

$$
\dot{\phi}_{i}=\frac{\alpha}{N} \sum_{l \in \mathcal{M}(m)}\left\{b_{l}^{e} m \sum_{q=1}^{l_{B}} k_{q}(i) \cos \left[l x_{q}(i)\right]+b_{l}^{o} m \sum_{q=1}^{l_{B}} k_{q}(i) \sin \left[l x_{q}(i)\right]\right\}
$$


where the numbers $k_{q}(i)$ and the angles $x_{q}(i)$ are as explained in Figure 2.3. In particular (as found in Ashwin and Swift [1992]), if $b_{l}^{e, o}=0$ for all $l \in \mathcal{M}(m)$, then the $l_{B}$-torus is a continuum of fixed points.

The vector field (2.15) may be calculated directly by plugging an arbitrary point on the invariant $l_{B}$-torus (i.e., with arbitrary $\left\{x_{1}(i), \ldots, x_{l_{B}}(i)\right\}$ for some $i$ ) into equation (2.2) and using the relationship discussed in connection with the proof of Proposition 2.7. The existence of continua of fixed points is obvious from equation (2.15). For odd $f$, the fixed tori may also be found by showing that the potential (2.4) is always constant under this same condition on the Fourier coefficients of $f$ given in Proposition 2.10.

If $m=N$ and $b_{l}^{e, o}=0$ for all $l=0(\bmod N), l_{B}=1$ and Proposition 2.10 simply gives the circle of equilibria that is the $T^{1}$ group orbit of the $Z_{N^{-}}$ symmetric equilibrium of Proposition 2.7 (with $k=1$ ). If $m=1$ then Proposition 2.10 gives no new information about fixed subspaces: $b_{l}^{e, o}=0$ for all $l \in \mathcal{M}(m)$ implies that the oscillators are uncoupled. Also, we note that since $S_{1} \times S_{1} \times \cdots \times S_{1} \subseteq S_{k_{1}} \times \cdots \times S_{k_{l_{B}}}$, the $l_{B}$-tori of fixed points guaranteed by the theorem are actually contained in the $(N / m)$ torus Fix $\left[\left(S_{1} \times S_{1} \times \cdots \times S_{1}\right)^{m} \rtimes Z_{m}\right]=\operatorname{Fix}\left[Z_{m}\right]$.

The following examples illustrate implications of Proposition 2.10 .

Example. Consider $N=4$ and suppose $b_{l}^{e, o}=0$ for even $l$. For $m=4$ the torus of fixed points guaranteed by the proposition is just the onetorus $\operatorname{Fix}\left[Z_{4}\right]$. For $m=2$, we get the two-torus of fixed points $\operatorname{Fix}\left[Z_{2}\right]$. This describes the set of points for which two oscillators are out of phase by $\pi$, and the other two are also out of phase by $\pi$, corresponding to $\left(\phi_{1}, \phi_{2}, \phi_{3}, \phi_{4}\right)=\left(\xi_{1}, \xi_{2}, \xi_{1}+\pi, \xi_{2}+\pi\right)$. Fix $\left[Z_{2}\right]$ contains both $\operatorname{Fix}\left[Z_{4}\right]=$ $\{(\xi, \xi+\pi / 2, \xi+\pi, \xi+3 \pi / 2)\}$ and $\operatorname{Fix}\left[\left(S_{2}\right)^{2} \rtimes Z_{2}\right]=\{(\xi, \xi, \xi+\pi, \xi+\pi)\}$.

$\operatorname{Fix}\left[Z_{2}\right]$ also coincides with the $(N-2=2)$-dimensional incoherent manifold' found for averaged arrays of Josephson junctions (Watanabe and Swift [1997]). The incoherent manifold is defined as the set with zero centroid of phases $\phi_{i}$ on the unit circle. Because Fix $\left[Z_{2}\right]$ is a fixed point subspace, the two-dimensional incoherent manifold is dynamically invariant as found in Watanabe and Swift [1997]; Proposition 2.10 gives conditions under which it is also dynamically fixed as well as the expression for drift along the manifold. Watanabe and Swift [1997] also show that the $(N-2)$ dimensional incoherent manifold is not dynamically invariant when $N \geq 5$.

However, this manifold contains dynamically invariant (and perhaps dynamically fixed) submanifolds: for $\phi$ in fixed point subspaces of isotropy subgroups which have $Z_{m}$ as a subgroup (where $m \geq 2$ ), the relevant centroid is zero. Thus, these fixed point subspaces are contained in the incoherent manifold. Note that the invariant (or fixed) tori have dimension $l_{B} \leq N / m$, which is less than $N-2$ for $N \geq 5, m \geq 2$.

Example. Suppose $N=6$ and $f(\cdot)=\sin (\cdot)$, and consider the $\left(\left(S_{3}\right)^{2} \rtimes\right.$ $Z_{2}$ )-invariant equilibria (e.g., $\left.\left(\phi_{1}, \phi_{2}, \phi_{3}, \phi_{4}, \phi_{5}, \phi_{6}\right)=(0,0,0, \pi, \pi, \pi) \equiv \bar{\phi}\right)$. 
From Proposition 2.7, the eigenvalues for such equilibria are 0 with multiplicity five, and $6 \alpha$ with multiplicity one. The null eigenvectors may be taken to be $e_{1}=(1,1,1,1,1,1), e_{2}=(2,-1,-1,0,0,0), e_{3}=(1,1,-2,0,0,0)$, $e_{4}=(2,-1,-1,2,-1,-1), e_{5}=(1,1,-2,1,1,-2)$. Figure 2.4 shows the potential $V$ corresponding to perturbations to $\bar{\phi}$ in the directions of these null eigenvectors. $V$ is flat for perturbations in the $e_{1}, e_{4}$, and $e_{5}$ directions (each with a corresponding one-dimensional continuum of fixed points, overall giving a three-torus of equilibria), but not for perturbations in the $e_{2}$ and $e_{3}$ directions. Proposition 2.10 guarantees the existence of the threetorus of equilibria $\operatorname{Fix}\left[Z_{2}\right]$ given by $\left(\phi_{1}, \phi_{2}, \phi_{3}, \phi_{4}, \phi_{5}, \phi_{6}\right)=\left(\xi_{1}, \xi_{2}, \xi_{3}, \xi_{1}+\right.$ $\left.\pi, \xi_{2}+\pi, \xi_{3}+\pi\right)$; note that perturbations to $\bar{\phi}$ in the $e_{1}, e_{4}$, and $e_{5}$ directions keep the system in the Fix $\left[Z_{2}\right]$ subspace. The $e_{2}$ and $e_{3}$ perturbations illustrate that every zero eigenvalue of Propositions 2.7 or 2.8 does not necessarily imply a corresponding one-dimensional continuum of fixed points.
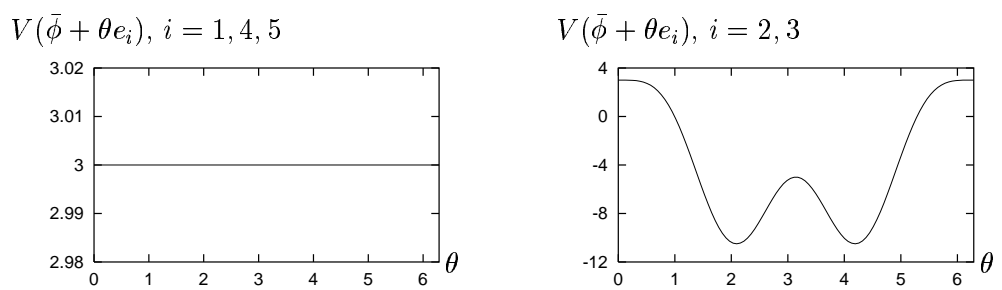

Figure 2.4. Potential $V$ for perturbations to $\bar{\phi}$ in the directions of the null eigenvectors, as defined in the text.

\section{Breaking the $T^{1}$ Symmetry: Product Coupling}

Reintroducing the $h$ and $g$ terms and going back to $\theta$ coordinates, we return to the $S_{N}$-equivariant system

$$
\dot{\theta}_{i}=\omega+\frac{\alpha}{N} \sum_{j=1}^{N} f\left(\theta_{j}-\theta_{i}\right)+h\left(\theta_{i}\right) \frac{\beta}{N-1} \sum_{j \neq i}^{N} g\left(\theta_{j}\right) .
$$

The results of this section are valid for arbitrary $C^{1} 2 \pi$-periodic functions $g$ and $h$; without loss of generality, we assume that $g$ takes values in $[0,1]$. Additional assumptions on the product function $G(\theta) \triangleq h(\theta) g(\theta)$ simplify the discussion of bifurcations in Section 3.1. 


\subsection{Bifurcations of fixed points on the diagonal $\operatorname{Fix}\left[S_{N}\right]$}

This section concerns analysis local to the diagonal of $\mathrm{T}^{N}$, defined by $\mathcal{D} \triangleq$ $\left\{\theta \mid \theta_{i}=\theta_{j} \forall i, j\right\}$, which is dynamically invariant. Restricted to $\mathcal{D}$ and with $\theta_{i} \equiv \theta$, equation (3.1) becomes

$$
\dot{\theta}=\omega+\alpha f(0)+\beta G(\theta) .
$$

This equation has fixed points given by $\bar{\theta}=G^{-1}\left(-\frac{\omega+\alpha f(0)}{\beta}\right)$. To simplify the analysis in this section, we assume that $G$ has a single minimum $\theta_{\text {min }}$ with $G^{\prime \prime}\left(\theta_{\min }\right) \neq 0$, as it does for the 'neurobiological' coupling functions to be considered in Section 4.

These conditions on $G$ and $\omega>0$ imply that there are no, one, or two ondiagonal fixed points, in the latter case denoted by $\bar{\theta}_{1}<\bar{\theta}_{2}$. The eigenvalues of the Jacobian of equation (3.1) linearized about these fixed points are

$$
\begin{array}{ll}
\lambda_{1}^{k}=-\alpha f^{\prime}(0)+\beta\left(h^{\prime}\left(\bar{\theta}_{k}\right) g\left(\bar{\theta}_{k}\right)-\frac{1}{N-1} h\left(\theta_{k}\right) g^{\prime}\left(\theta_{k}\right)\right), & \text { multiplicity } N-1, \\
\lambda_{2}^{k}=\beta G^{\prime}\left(\bar{\theta}_{k}\right), & \text { multiplicity } 1 .
\end{array}
$$

Stability in the transverse directions (with respect to the diagonal) is determined by $\lambda_{1}$, and in the axial direction by $\lambda_{2}$; note that our hypothesis on $G$ implies that $\lambda_{2}\left(\bar{\theta}_{1}\right)<0$, and $\lambda_{2}\left(\bar{\theta}_{2}\right)>0$. As $\beta$ is decreased through $\beta=(\omega+\alpha f(0)) /\left|G\left(\theta_{\text {min }}\right)\right|$, the two fixed points coalesce and disappear in a saddle node bifurcation (the appearance of these fixed points as $\beta$ increases represents the phenomenon of oscillator death: Ermentrout and Kopell [1990]; Taylor and Holmes [1998]). For the remaining values of $\beta$, the orbit along $\mathcal{D}$ is the $\left(S_{N}\right.$ symmetric) periodic orbit $\theta_{D}(t)$. We will investigate the period and stability of this orbit in the following sections.

\subsection{Frequency and stability of the in-phase periodic orbit}

If $\beta<(\omega+\alpha f(0)) /\left|G\left(\theta_{\text {min }}\right)\right|$, the period of the orbit on $\mathcal{D}$ is given by

$$
\tau=\int_{0}^{2 \pi}\left(\frac{d \theta}{d t}\right)^{-1} d \theta=\int_{0}^{2 \pi} \frac{d \theta}{\omega+\alpha f(0)+\beta h(\theta) g(\theta)} .
$$

Moreover, we have

3.1 Proposition. (Local stability of $\mathcal{D}$.) The $S_{N}$-symmetric periodic solution $\theta_{i}(t) \equiv \theta_{D}(t)$ along $\mathcal{D}$ is asymptotically stable if

$$
\alpha>\frac{\beta N}{(N-1) \tau f^{\prime}(0)} \int_{0}^{2 \pi} \frac{g(\theta) h^{\prime}(\theta)}{\omega+\alpha f(0)+\beta g(\theta) h(\theta)} d \theta,
$$

where $\tau$ is the (generally $\alpha$-dependent) period of $\theta_{D}(t)$ given in equation (3.3) and we assume $f^{\prime}(0)>0$. 
Closely related results are found in Tsang, Mirollo, and Strogatz [1991]; Golomb, Hansel, Shraiman, and Sompolinsky [1992].

Proof. Linearized around $\theta_{D}(t)$, equation (3.1) becomes $\dot{\xi}_{i}=\left[A_{d}(t) \xi\right]_{i}$. The proof uses the fact that the ( $t$-dependent) symmetric matrix $A_{d}(t)$ has a particularly simple structure, and that it can be diagonalized by a $t$-independent similarity transformation. Specifically, the eigenvalues of $A_{d}(t)$, where $t$ is viewed as a (fixed) parameter, are:

$$
\begin{aligned}
& \lambda_{1}(t)=-\alpha f^{\prime}(0)+\beta\left(g(t) h^{\prime}(t)-\frac{1}{N-1} h(t) g^{\prime}(t)\right), \quad \text { multiplicity } N-1, \\
& \lambda_{2}(t)=\beta G^{\prime}(t), \quad \text { multiplicity } 1,
\end{aligned}
$$

where $g(t)$ is written for $g\left(\theta_{D}(t)\right)$, etc. The orthogonal eigenvectors of $\lambda_{1}(t)$ (denoted by $\chi_{1}, \ldots, \chi_{N-1}$ ) may be chosen constant and orthogonal to the eigenspace of $\lambda_{2}(t)$, which is spanned by the eigenvector $(1, \ldots, 1)^{\mathrm{T}}$. Thus, $\chi_{1}, \ldots, \chi_{N-1}$ span the space normal to $\theta_{D}(t)$. In these eigencoordinates the linearized system decouples as

$$
\dot{\xi}_{i}=\lambda_{1}(t) \xi_{i}, \quad i=1, \ldots, N-1, \quad \dot{\xi}_{N}=\lambda_{2}(t) \xi_{N} .
$$

Define the $(N-1)$-dimensional plane $\sum=\left\{\chi \mid \chi_{N}=0\right\}$ and consider the Poincaré map $P: U \rightarrow U$ for some neighborhood $U \subset \sum$ of 0 . The orbit $\theta_{D}(t)$ intersects $\sum$ at 0 , which is a fixed point for $P$. For $i=1, \ldots, N-$ $1, P: \xi_{i} \mapsto\left(\exp \int_{0}^{\tau} \lambda_{1}(t) d t\right) \xi_{i}$ so that 0 is a stable fixed point for $P$ if $\int_{0}^{\tau} \lambda_{1}(t) d t<0$. (Due to the periodicity of $G(t), \int_{0}^{\tau} \lambda_{2}(t) d t \equiv 0$, as it must, being the Floquet exponent along the periodic orbit). We have

$$
\begin{aligned}
\int_{0}^{\tau} & \lambda_{1}(t) d t \\
= & \int_{0}^{\tau}\left(-\alpha f^{\prime}(0)+\beta\left[g(t) h^{\prime}(t)-\frac{1}{N-1} h(t) g^{\prime}(t)\right]\right) d t \\
= & -\alpha f^{\prime}(0) \tau+\int_{0}^{2 \pi} \beta\left(-\frac{\left[h(\theta) g^{\prime}(\theta)+h^{\prime}(\theta) g(\theta)\right]}{N-1}+\frac{N g(\theta) h^{\prime}(\theta)}{N-1}\right) \dot{\theta}^{-1} d \theta \\
= & -\alpha f^{\prime}(0) \tau-\frac{1}{N-1} \ln [\omega+\alpha f(0)+\beta h(\theta) g(\theta)]_{0}^{2 \pi} \\
& +\int_{0}^{2 \pi} \frac{\beta N g(\theta) h^{\prime}(\theta)}{(N-1)(\omega+\alpha f(0)+\beta h(\theta) g(\theta))} d \theta \\
= & -\alpha f^{\prime}(0) \tau+\int_{0}^{2 \pi} \frac{\beta N g(\theta) h^{\prime}(\theta)}{(N-1)(\omega+\alpha f(0)+\beta h(\theta) g(\theta))} d \theta,
\end{aligned}
$$

where the second term in equation (3.5) vanishes due to the $2 \pi$-periodicity of $h$ and $g$. Thus, $\int_{0}^{\tau} \lambda_{1}(t) d t<0$ when the inequality of Proposition 3.1 is satisfied. Since stability of the fixed point 0 under $P$ implies stability of $\theta_{D}(t)$ for equation (2.1), the Proposition is proven. 
A simple calculation using integration by parts and the $2 \pi$-periodicity of $g$ and $h$ shows that for $\alpha=0$ and asymptotically small $\beta$, the right-hand side of (3.6) becomes $\frac{\beta N}{N-1} f_{s}^{\prime}(0)$, which (cf. (2.13)) determines the stability of the in-phase solution if synaptic coupling $\frac{\beta}{N-1} h\left(\theta_{i}\right) \sum_{j \neq i} g\left(\theta_{j}\right)$ is taken to be weak and then averaged to yield $\frac{\beta}{N-1} \sum_{j \neq i} f_{s}\left(\theta_{j}-\theta_{i}\right)$. This agreement between the averaged and original versions of (3.1) for sufficiently small $\beta$ is expected from the averaging theorem (Guckenheimer and Holmes [1983]), and reveals how (3.4) generalizes the stability result found in van Vreeswijk, Abbot, and Ermentrout [1994]; Hansel, Mato, and Meunier [1995] for $N=2$ and averaged synaptic coupling.

Equation (3.4) may be used to estimate a critical value $\alpha_{l o c}$ such that $\theta_{D}(t)$ is asymptotically stable for $\alpha>\alpha_{l o c}$. Letting $\widehat{h}$ be a Lipschitz constant for $h$, we note that

$$
\int_{0}^{2 \pi} \frac{g(\theta) h^{\prime}(\theta)}{\omega+\alpha f(0)+\beta h(\theta) g(\theta)} d \theta \leq \int_{0}^{2 \pi} \frac{\widehat{h}}{\omega+\alpha f(0)+\beta h(\theta) g(\theta)} d \theta=\widehat{h} \tau,
$$

where the inequality follows from the bound on $g$ and the definition of the Lipschitz constant. Thus, from (3.4) we have stability if $\alpha>\frac{N \beta \widehat{h}}{(N-1) f^{\prime}(0)} \triangleq$ $\alpha_{l o c}$. With $f(0)=0$ (e.g. if $f$ is odd), this estimate can be refined: the right-hand side of equation (3.4) is independent of $\alpha$, so that the (smallest) critical value $\tilde{\alpha}_{l o c}$ is

$$
\tilde{\alpha}_{l o c}=\frac{\beta N}{(N-1) \tau f^{\prime}(0)} \int_{0}^{2 \pi} \frac{g(\theta) h^{\prime}(\theta)}{\omega+\beta g(\theta) h(\theta)} d \theta .
$$

We now turn to the nonlinear stability properties of $\mathcal{D}$.

\section{Estimate for the domain of attraction of $\mathcal{D}$}

3.2 Proposition. (Nonlinear stability of $\mathcal{D}$.) For some $s>1$, assume $f^{\prime}(0)>0$ and let $R>0$ be the smallest value for which either $f^{\prime}(2 R)=$ $f^{\prime}(0) / s>0$ or $f^{\prime}(-2 R)=f^{\prime}(0) / s>0$ (implying $\min _{\theta \in[-2 R, 2 R]} f^{\prime}(\theta)=$ $\left.f^{\prime}(0) / s\right)$. Let $\widehat{G}$ be the Lipschitz constant for $G(\cdot)=g(\cdot) h(\cdot)$, and define $\widehat{h}_{1}\left(\theta_{i}\right)=\max _{\theta}\left\{\left|h^{\prime}\left(\theta_{i}\right) g(\theta)\right|:\left|\theta_{i}-\theta\right|<2 R\right\}$ and $\widehat{h}_{1}=\max _{\theta_{i}}\left\{\widehat{h}_{1}\left(\theta_{i}\right)\right\}$. Then, for

$$
\alpha>\alpha_{g l o b} \triangleq \frac{s \beta\left(N \widehat{h}_{1}+\widehat{G}\right)}{(N-1) f^{\prime}(0)},
$$

the domain of attraction for $\mathcal{D}$ includes $\mathcal{C}_{R} \triangleq\left\{\theta \mid d\left(\theta, \theta_{d}(\psi)\right) \leq R\right.$ for some $\psi \in[0,2 \pi]\}$ (cf. Figure 2.2).

Proof. Fix an arbitrary $\psi \in[0,2 \pi)$. Consider the (non-orthogonal) basis $b \equiv\left\{x_{i} \mid i=1, \ldots, N-1\right\}$, where $x_{i} \equiv \theta_{i}-\theta_{i+1}$. We define $X_{\psi}$, the $N-1$ 
dimensional space perpendicular to the axis of $\mathcal{C}_{R}$ at $\theta_{d}(\psi)$, as the copy of span $b$ containing $\theta_{d}(\psi)$. In other words, $X_{\psi}$ is the normal space $\mathbf{N}\left(\theta_{d}(\psi)\right)$.

Now, define the squared 'radius' $\mathcal{R}=\sum_{i=0}^{N-1} x_{i}^{2}$. We will show that $\dot{\mathcal{R}}=$ $2 \sum_{i=0}^{N-1} x_{i} \dot{x}_{i} \leq 0$ for all $\mathbf{x} \in \mathcal{C}_{R}$. The cylindrical surfaces $\{\mathbf{x} \mid \mathcal{R}(\mathbf{x})=c\}$ will therefore be crossed 'inward' toward the axis of $\mathcal{C}_{R}$.

Take an arbitrary $\theta \in \mathcal{C}_{R} \cap X_{\psi}$. For such a $\theta$, we also have $\theta \in B_{R}(\psi)=$ $\left\{\theta \mid d\left(\theta, \theta_{d}(\psi)\right)<R\right\}$. Thus $\left|\theta_{j}-\theta_{i}\right|<2 R \forall i, j$ (and, in particular, $\left|x_{i}\right|<$ $2 R \forall i)$. These inequalities allow us to find a bound on each $\dot{x}_{i}$ :

$$
\left.\begin{array}{rl}
\dot{x}_{i}= & \overline{\theta_{i}-\theta_{i+1}} \\
= & \frac{\alpha}{N} \sum_{j=1}^{N} f\left(\theta_{j}-\theta_{i}\right)-\frac{\alpha}{N} \sum_{j=1}^{N} f\left(\theta_{j}-\theta_{i+1}\right) \\
& \quad+\frac{\beta}{N-1} h\left(\theta_{i}\right) \sum_{j \neq i}^{N} g\left(\theta_{j}\right)-\frac{\beta}{N-1} h\left(\theta_{i+1}\right) \sum_{j \neq i+1}^{N} g\left(\theta_{j}\right) \\
= & \frac{\alpha}{N} \sum_{j=1}^{N}\left[f\left(\theta_{j}-\theta_{i}\right)-f\left(\theta_{j}-\theta_{i}+x_{i}\right)\right]+\frac{\beta\left[h\left(\theta_{i}\right)-h\left(\theta_{i+1}\right)\right]}{N-1} \sum_{j=1}^{N} g\left(\theta_{j}\right) \\
& +\frac{\beta\left[h\left(\theta_{i+1}\right) g\left(\theta_{i+1}\right)-h\left(\theta_{i}\right) g\left(\theta_{i}\right)\right]}{N-1} . \\
\dot{x}_{i} & <-\alpha\left[f^{\prime}(0) / s\right] x_{i}+\beta \frac{N}{N-1} \widehat{h}_{1} x_{i}+\frac{\beta}{N-1} \widehat{G} x_{i} \triangleq k x_{i} \quad \text { if } x_{i}>0 \\
>-\alpha\left[f^{\prime}(0) / s\right] x_{i}+\beta \frac{N}{N-1} \widehat{h}_{1} x_{i}+\frac{\beta}{N-1} \widehat{G} x_{i} \triangleq k x_{i} \quad \text { if } x_{i}<0
\end{array}\right\} .(3.11)
$$

The inequalities (3.11) use the hypothesis on $f^{\prime}$, the bound $g(\theta) \leq 1$, and the definitions of $\widehat{h}$ and $\widehat{G}$. Thus, for $k<0$ (i.e. $\alpha>\alpha_{\text {glob }}$ ), $\dot{\mathcal{R}}=$ $2 \sum_{i=0}^{N-1} x_{i} \dot{x}_{i}<0$ unless $x_{i}=0, \forall i$. This argument may be repeated for any $\psi$ and therefore for any arbitrary $\theta \in \mathcal{C}_{R}$, so the Proposition follows.

Since nonlinear stability implies local stability, it must follow from $\alpha>$ $\alpha_{g l o b}$ that inequality (3.4) is satisfied. This may be seen from the fact that $\alpha>\alpha_{g l o b}$ implies $\alpha>\alpha_{l o c}$ and comparing equation (3.9) with (3.7).

Finally, we note that Proposition 3.2 may be sharpened by refining the estimates in (3.11) in any manner that also implies $\operatorname{sign}\left(\dot{x}_{i}\right)=-\operatorname{sign}\left(x_{i}\right)$. For example, a lower value $\widehat{h}_{2}$ can replace $\widehat{h}_{1}$ above, where $\widehat{h}_{2}=\max _{\theta_{i}} \widehat{h}_{2}\left(\theta_{i}\right)$ and $\widehat{h}_{2}\left(\theta_{i}\right)=\max _{\theta}\left\{h^{\prime}\left(\theta_{i}\right) g(\theta):\left|\theta_{i}-\theta\right|<2 R\right\}$ (note that although we have dropped the absolute value in the $\max _{\theta}, \widehat{h}_{2} \geq 0$ since $h$ is periodic). The bound $\widehat{h}_{2}$ arises as follows. If the second term in (3.10) is of opposite sign to $x_{i}$, it favors the nclusion $\operatorname{sign}\left(\dot{x}_{i}\right)=-\operatorname{sign}\left(x_{i}\right)$ and hence may be ignored for the purposes of bounding $\alpha$ such that $k<0$. Thus the natural question is: assuming that it is of the same sign as $x_{i}$, can we find a smaller upper bound than $\beta \frac{N}{N-1} \widehat{h}_{1} x_{i}$ on the magnitude of this second term? The answer 
is yes: since $\left[h\left(\theta_{i}\right)-h\left(\theta_{i+1}\right)\right]=\left[h\left(x_{i}+\theta_{i+1}\right)-h\left(\theta_{i+1}\right)\right]$, this difference cannot exceed the upper bound $\beta \frac{N}{N-1} \widehat{h}_{2} x_{i}$, as desired.

\section{Application to a model of the locus coeruleus}

Here we apply the analysis above to a model of the locus coeruleus brain nucleus. First, we introduce specific coupling functions $f, g$, and $h$ appropriate to neuronal coupling.

\subsection{Coupling functions}

The functions $f$ and $g, h$, corresponding to electrotonic and synaptic coupling, were computed using both the strong attraction (SA) and phase response (PR) methods mentioned in the introduction. The Hodgkin-Huxley (HH) equations with input current $10 \mu \mathrm{A} / \mathrm{cm}^{2}$ were used (Hodgkin and Huxley [1952]). In their original form these equations were derived from the giant axon of a squid, so their use here merely represents a proof of concept. Reduction of more realistic mammalian neuron models, which include calcium-dependent potassium channels and whose action potential spikes occupy a much smaller fraction of the period than in the rescaled $\mathrm{HH}$ equations, is in progress (Brown, Moehlis, Holmes, Aston-Jones, and Clayton [2002]), and leads to coupling functions somewhat different from those considered here, although the general structure of the phase equations survives.

The effect of electrotonic coupling on the time derivative $\dot{V}_{i}$ of neuron $i$ 's voltage of was taken to be $\frac{\alpha}{N} \sum_{j=1}^{N}\left(V_{j}-V_{i}\right)$ (cf. Johnston and Wu [1997]), and the inhibitory synaptic effect to be $\left(E_{K}-V_{i}\right) \frac{\beta}{N-1} \sum_{j \neq i} A\left(V_{j}, t\right)$, where $E_{K}$ is the reversal potential for potassium and $A\left(V_{j}, t\right)$ is an 'alpha function' which takes values in $[0, \tilde{A}], \tilde{A}<1$, and represents the influence of neuron $j$ on post-synaptic cells. Specifically, $A\left(V_{j}, t\right)=\left(\left(t-t_{s}^{j}-t_{d}\right) / \tau_{A}\right) \cdot \exp (-(t-$ $\left.\left.t_{s}^{j}-t_{d}\right) / \tau_{A}\right)$, where $t_{s}^{j}$ is the time at which the voltage of neuron $j$ spikes (see below), $t_{d}$ is the synaptic delay, and $\tau_{A}$ is the synaptic time constant (e.g. Kim and Lee [2000]). The effective value of $\tau_{A}$ for $\mathrm{LC}$ neurons has been observed to be much longer than those of typical synaptic connections due to the slow dynamics of norepinephrine neurotransmitter uptake (Grant, Aston-Jones, and Redmond [1988]; Aston-Jones, Rajkowski, and Alexinsky [1994]). We parameterize the limit cycle of the uncoupled HH equations by a time scaled so that the period $T=\frac{2 \pi}{\omega}$ is $1 / 3 \mathrm{sec}$ (to match our estimate for LC neurons), and take $\tau_{A}=0.025 \mathrm{sec}$ and $t_{d}=0.150 \mathrm{sec}$. These neuron and coupling models and parameters lead to the reductions of the coupling functions to $\mathrm{T}^{N}$ displayed in Figure 4.1. 

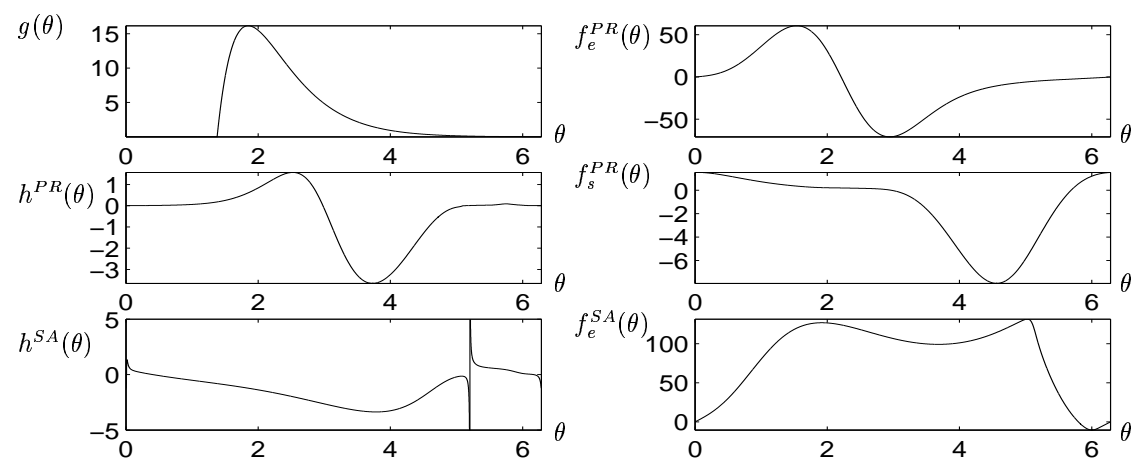

Figure 4.1. Coupling functions derived from the (time-reparameterized) Hodgkin-Huxley equations. The subscript $e(s)$ refers to electrotonic (synaptic) coupling, while the superscript $P R(S A)$ indicates that the function was derived using the phase reduction (strong attraction) method. The $h$ 's and $g$ are calculated for the synaptic coupling described in the text: $f_{s}^{P R}$ is obtained by averaging the product of $g$ and $h^{P R}$, and $f_{e}^{P R}$ is obtained by averaging the electrotonic coupling using a phase response method (cf. Kim and Lee [2000]). $f_{e}^{S A}$ is obtained by first assuming that the limit cycle is infinitely attracting, followed by averaging (cf. Ermentrout and Kopell [1990]; Hoppensteadt and Izhikevich [1997]). The 'spikes' in $h^{S A}(\theta)$ are associated with the projection of coupling functions near turning points in the original phase variables; the tips extend to approximately \pm 30 (there is also an $\mathcal{O}(1)$ spike near $\theta=0$, not visible here).

Under the idealisation that neurons in the small LC nucleus are identical (Williams, North, Shefner, Nishi, and Egan [1984]) and globally coupled, our LC model simplifies to equation (1.2). As coupling becomes stronger, modifications to $f, g$, and $h$ may be required to maintain the accuracy of phase reductions; for the purpose of this paper, these effects are neglected. However, Brown, Moehlis, Holmes, Aston-Jones, and Clayton [2002] includes a careful comparison of phase-reduced and 'full' conductance-based $\mathrm{LC}$ models in the relevant parameter range.

\subsection{Modeling synchrony in LC modes}

This section demonstrates that cross correlograms qualitatively similar to those of Figure 1.1(a) can arise in our phase-reduced LC model due to increased coupling in the phasic LC mode relative to the tonic mode.

Cross correlograms are derived from solutions of (3.1) as follows. A spike is deemed to occur when a rotator $\theta_{i}$ crosses through a threshold value $\theta_{s}$ : the solution of $\left\{V\left(\theta_{s}\right)=V_{s}, V^{\prime}\left(\theta_{s}\right)>0\right\}$, where $V_{s}=-30 m V$ is a depolarized voltage characteristic of a neuron firing an action potential and the function $V(\theta)$ is defined by $V(t)=V(\theta / \omega)$ over the period of one neuron action potential. The set of all pairwise differences between times at 
which distinct spike events occur is computed according to this definition, and the cross correlogram is the histogram of this set.

\section{Phase-difference coupling}

\begin{tabular}{|c|c|c|c|c|c|}
\hline & $\begin{array}{c}\mathrm{PR} \\
\kappa=0\end{array}$ & $\begin{array}{c}\mathrm{PR} \\
\kappa=10\end{array}$ & $\begin{array}{c}\mathrm{PR} \\
\kappa=50\end{array}$ & $\begin{array}{c}\mathrm{PR} \\
\kappa=100\end{array}$ & $\begin{array}{c}\mathrm{SA} \\
\kappa=0\end{array}$ \\
\hline$\left(\mathbf{S}_{\mathbf{k}}\right)^{\mathbf{m}} \rtimes \mathbf{Z}_{\mathbf{m}}$ & & & & & \\
\hline $\mathrm{S}, m=$ & $1,2,4$ & $1,2,4$ & 1,4 & 1 & $1,3,4$ \\
\hline $\mathrm{U}, m=$ & $3,6,8,12$ & $3,6,8,12$ & $2,3,6,8,12$ & $2,3,4,6,8,12$ & $2,6,8,12$ \\
\hline $\mathbf{S}_{\mathbf{N}-\mathbf{p}} \times \mathbf{S}_{\mathbf{p}}$ & & & & & \\
\hline $\mathrm{S}, p=$ & 11,12 & 12 & 1,2 & $1-4$ & $1-6$ \\
\hline $\mathrm{U}, p=$ & $1-12$ & $1-12$ & $1-12$ & $3-12$ & $7-12$ \\
\hline
\end{tabular}

TABLE 4.1. The linear stability of various 'clustered' periodic orbits of diagonal flow for different phase difference couplings $f_{e}\left(\theta_{j i}\right)+\kappa f_{s}\left(\theta_{j i}\right)$, computed using the PR or SA methods. $\left(S_{k}\right)^{m} \rtimes Z_{m}$ stability is given for allowable $m(N=24)$, and $S_{N-p} \times S_{p}$ stability for $N=24$ and $p=1, \ldots, 12$; a value of $p$ being listed twice indicates the correspondence of multiple $\delta$ 's. S and U indicate asymptotic stability and instability, respectively, for the $m$ or $p$ values given in the subsequent columns.

In this section we assume that synaptic coupling $\frac{\beta}{N-1} h\left(\theta_{i}\right) \sum_{j \neq i} g\left(\theta_{j}\right)$ is sufficiently weak that it can be averaged to yield $\frac{\beta}{N-1} \sum_{j \neq i} f_{s}\left(\theta_{j}-\theta_{i}\right)$, and we take $\beta=\kappa \alpha$, so that (3.1) becomes $\dot{\theta}_{i}=\omega+\frac{\alpha}{N} \sum_{j} f\left(\theta_{j i}\right)$, where the phase difference function $f(\cdot)=f_{e}(\cdot)+\kappa f_{s}(\cdot)$. We use the methods of Section 2 to determine stability of periodic orbits. The results are shown in Table 4.1 for various values of $\kappa$ and coupling functions derived with the PR and SA methods. The SA coupling functions give rise to a larger set of distinct stable periodic orbits, the consequences of which will be discussed below.

To model the phasic and tonic behavior of Figure 1.1, we consider equation (2.1) in the presence of noise represented as additive Brownian forcing on the torus, so that:

$$
d \theta_{i}=\left[\omega+\frac{\alpha}{N} \sum_{j \neq i}^{n} f\left(\theta_{j}-\theta_{i}\right)\right] d t+\sigma d W_{t}^{i}
$$

The inclusion of random noise represents additional inputs currents to LC neurons, a common stratagem in accounting for the influence of neural subgroups neglected in the model. The stochastic averaging theorem (Zhu [1988]; Freidlin and Wentzell [1998]) leads to the approximation above, including $\sigma$ being independent of $\theta$; in particular, $\sigma^{2}=\overline{z^{2}} \gamma$, where $\gamma$ is the variance of Brownian input currents, $z(\theta)$ is the phase response curve (PRC, cf. Kuramoto [1997]), and the overbar denotes the average 
$\overline{(\cdot)}=\frac{1}{2 \pi} \int_{0}^{2 \pi}(\cdot) d \theta$. Simulations of equation (4.1) to be discussed below were performed using a second order stochastic Runge-Kutta method (Honeycutt [1992]).

The stability results of Table 4.1 will persist for $\alpha$ sufficiently larger than $\sigma$, but solutions approach a constant-drift Brownian motion on $\mathrm{T}^{N}$ as the coupling/noise ratio $\alpha / \sigma$ decreases. These two regimes correspond to the biological hypothesis discussed in the Introduction: that tonic behavior can be expected for relatively weak coupling $\left(\alpha_{t o n}\right)$ and phasic behavior for larger $\alpha_{p h}$. For $\alpha_{p h}$, the presence of appropriate stable states can lead to the phasic-mode cross correlogram via several mechanisms (see below and Figures 4.2(b-e)). Meanwhile, for the lesser value of $\alpha_{t o n}$, the essentially constant-drift Brownian motion on the torus leads to the flat cross correlogram characteristic of this mode (i.e., no preferred firing time difference between neurons); see Figure 4.2(a).

If a variety of diagonal flow solutions are simultaneously stable (as for the SA coupling functions) the following mechanism can produce the "peakshoulder' cross correlogram patterns characteristic of the phasic LC mode (Figure 1.1(a)). For every revolution (of diagonal flow), $\left(S_{k_{1}} \times S_{k_{2}}\right)^{m} \rtimes Z_{m}$ states with $\delta \neq 0, \pi$ produce cross correlograms with $m\left(k_{1}^{2}+k_{2}^{2}-k_{1}-k_{2}\right)$ counts at $t=0, m\left(k_{1}^{2}+k_{2}^{2}\right)$ counts at times proportional to $\pm 2 \pi j / m$, $j=1, \ldots, m-1$, and $m k_{1} k_{2}$ counts at times proportional to $\pm(2 \pi j / m) \pm \delta$, $j=0, \ldots, m-1$. If $N \geq 5$, this leads to a dominant central peak in the cross correlogram for (two-cluster) states with $m=1$. Moreover, all peaks except for the central peak at 0 will be differently spaced for each distinct $\left(S_{k_{1}} \times S_{k_{2}}\right)^{m} \rtimes Z_{m}$ orbit. Thus, if the individual cross correlograms from many of these states are combined (e.g. due to stochastic switching due to random noise in (4.1)), the common central maxima would conspire to produce a central peak in the cross correlogram while the combination of many secondary maxima could give rise to the relatively flat shoulder. This is demonstrated in Figure 4.2(c).

However, the stochastic switching mechanism does not apply to the PR functions, which exhibit relatively few stable periodic orbits significantly different from the in-phase mode (in particular, $\delta<1$ for the stable $S_{p} \times$ $S_{N-p}$ modes for all $\kappa$ values in Table 4.1). For the PR functions, then, the shoulders in the cross correlogram instead arise when the stable distribution around the in-phase equilibrium is broad; this was found (Figure 4.2(b)) to require high noise strengths, approximately $\sigma>\alpha \max _{\theta}\left\{\left|f_{e}(\theta)+\kappa f_{s}(\theta)\right|\right\} / 3$. Faced with this perhaps unrealistically high value, in the next section we explore the presence of random natural frequencies as another method for creating the cross correlogram shoulders.

To check the validity of the SA and PR phase reductions, we compared a few cases of our stability predictions with numerical simulations of the full HH equations (cf. Kim and Lee [2000]); for $\kappa=0$, stability was consistent with the full equations for the PR reductions but not for SA. Thus, all 
subsequent computations are given for the PR case; however, to illustrate properties of (2.1) we will continue to refer to the SA functions when their general form gives additional (e.g. contrasting) results.
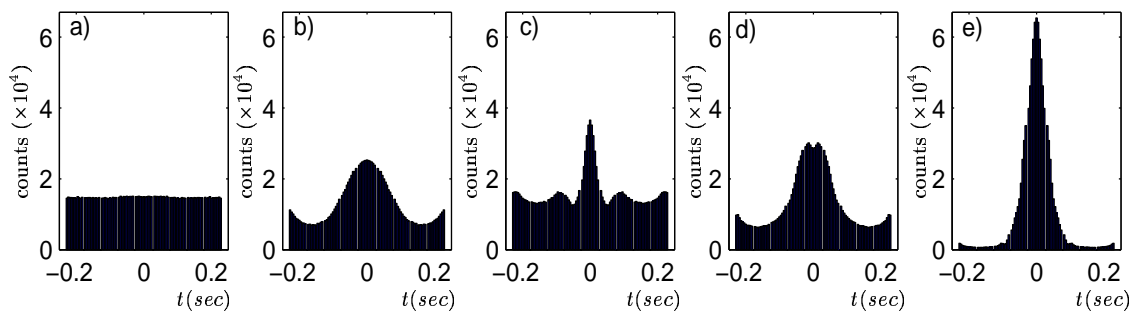

Figure 4.2. Cross correlograms for simulations of the tonic 'low-coupling' (a) and phasic 'high-coupling' (b-e) LC modes using the phase-difference model (4.1). To facilitate comparison, $\alpha_{p h}$ was chosen so that $\alpha_{p h} \max _{\theta}\{|f(\theta)|\}=2.25$ for both the SA and PR coupling functions; also, $\alpha_{t o n}=\alpha_{p h} / 5, \omega=6 \pi$, and $N=24$. (a) PR model with $\kappa=0, \sigma=0.8$, and $\alpha=\alpha_{\text {ton }}$; (b) same except with $\alpha=\alpha_{p h}$; (c) SA model with $\kappa=0$ and $\sigma=0.2, \alpha=\alpha_{p h}$. (d) As described in Section 4.2, phasic PR simulation as in (a) but with randomly distributed frequencies (drawn from the Cauchy distribution with parameter 0.2 and mean $\omega$ ) and $\sigma=0.1$. (e) as in (b), but with the addition of phase-dependent (synaptic) coupling of strength $\beta=0.23$. The range of all histograms is $[-.7 \tau, .7 \tau]$, where $\tau=1 / 3 \mathrm{sec}$ is the simulated natural period; all histograms are averaged over five simulated recordings with uniformly distributed initial conditions, with each trial 2 min in duration.

\section{Breaking the $S_{N}$ symmetry with random natural frequencies}

By the standard theorems for normally hyperbolic invariant manifolds Fenichel [1971]; Wiggins [1994], many of our stability results persist under small perturbations from fully $S_{N} \times T^{1}$ - or $S_{N}$-equivariant systems. To study these effects, we performed numerical simulations: in particular, we considered the phase-difference system (4.1) with randomly distributed frequencies $\omega \rightarrow \omega_{i}$ (so that the noise-free system has no nontrivial isotropy subgroups). This is appropriate because individual LC neurons exhibit a range of natural frequencies Grant, Aston-Jones, and Redmond [1988]; Aston-Jones, Rajkowski, and Alexinsky [1994]. In Figure 4.2(d), we show a reproduction of the phasic mode cross correlogram for the averaged PR coupling function with $\kappa=0$ and frequencies randomly distributed around $\omega$ with a Cauchy distribution with parameter 0.2 . This corresponds to a tight distribution; nevertheless, the relatively low noise value $\sigma=0.1$ is sufficient to reproduce patterns similar to those in Figure 1.1.

We note that there are some analytical results (e.g. Kuramoto [1984]; Strogatz [2000]; Crawford [1995]; Crawford and Davies [1999]) for the distributed frequency, phase-difference system as $N \rightarrow \infty$. However, a finite- $N$ 
analysis of the full equation (2.1) with distributed phases and phase product coupling term remains open, to the best of our knowledge.

\section{Phase-dependent coupling}

We now return to consider equation (3.1) with unaveraged synaptic (product) coupling. Since $f(0)=0$ for electrotonic coupling, we may use equation (3.8) to explicitly calculate a lower bound on $\alpha$ for this orbit to be stable. The resulting $\tilde{\alpha}_{l o c}<0$ (Figure 4.3 ), so that the in-phase state is stable for synaptic coupling and any positive $\alpha$ (since $f^{\prime}(0)>0$ ). In addition, the domain of attraction may be estimated: for example, taking $s=2.14$ in Proposition 3.2 gives $R=1 / 4$ and $\widehat{h}_{2}=28.1$ for PR coupling functions with $\kappa=0$. Thus, the domain of attraction of the in-phase orbit includes $\mathcal{C}_{R}$ if $\alpha>\alpha_{\text {glob }}=7.11 \beta$ (equation (3.9) for $N \rightarrow \infty$ ). Figure 4.2(e) demonstrates the collapse of the cross correlogram (d) upon addition of the synchronizing (cf. Section 4.2) product coupling.

For the SA coupling functions, the following observation is useful: since the denominator in the integrand of (3.4) is always positive, if $h^{\prime}(\theta)<0$ for $\theta$ in the (essential) support of the positive function $g$ then the integrand itself will always be negative, giving stability for any $\alpha>0$. The plots in Figure 4.1 show that for delays $t_{d}$ (which correspond to translations of $g$ ) taken in a wide range around $0.150 \mathrm{sec}$, stability holds for arbitrary $g$ of reasonably compact essential support and any $\alpha, \beta>0$ such that the inphase periodic orbit exists. We note here that related stability conditions are derived in van Vreeswijk, Abbot, and Ermentrout [1994]; Ermentrout [1996]; Gerstner, van Hemmen, and Cowan [1996], in which the time course of $g$ is also important.

\subsection{Modeling firing rates in LC modes}

Here we show that the depressed firing rates characteristic of the phasic mode can be captured by our LC model if explicitly phase-dependent synaptic terms are included.

\section{Phase-difference coupling}

Under the assumption that the period of the in-phase state largely determines average LC firing rates, the depressed firing rate observed in the phasic mode cannot be reproduced with averaged (phase-difference), PR coupling functions. The period of the in-phase state of $(4.1)$ is $\frac{2 \pi}{\omega+\alpha f(0)}$, and Figure 4.1 shows that $f(0)>0$ for any $\kappa>0$, so that the period will always decrease as $\alpha$ increases. Hence, the (highly-coupled) phasic state would actually have an increased firing rate. In contrast, we shall see that (3.1) allows us to correctly reproduce observed firing rates. 


\section{Phase-dependent coupling}

For small $\beta$, where averaging is valid, the period of the $S_{N}$ symmetric orbit must decrease with $\beta$. However, Figure 4.3 also shows that for $\beta$ sufficiently large, the period increases with $\beta$ (the term $G(\theta)$ in (3.2) slows the flow). Since the $S_{N}$ symmetric orbit is attracting, its increasing period indicates a mechanism for lower firing rates in the phasic mode. This shows the importance in this case of considering the explicit product coupling of (3.1); however, for other parameter values and neuron models phasedifference coupling may correctly capture trends in firing rates (cf. Brown, Moehlis, Holmes, Aston-Jones, and Clayton [2002]).

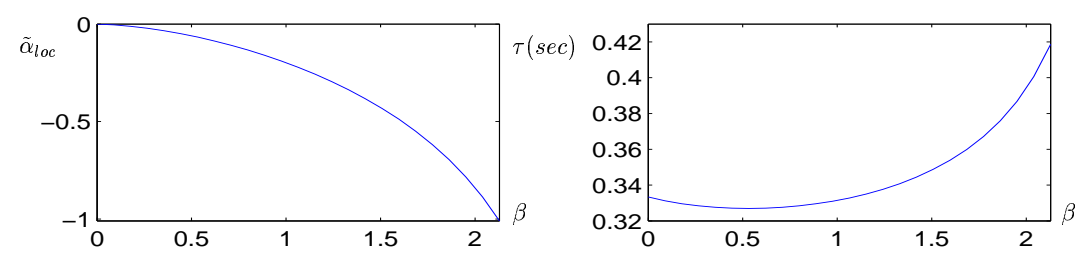

FiguRE 4.3. The $\beta$-dependence of (left) bounds for stability and (right) the period of the $S_{N}$ symmetric orbit with PR coupling functions, from equations (3.8) and (3.3) in the large- $N$ limit.

\subsection{LC response to stimulus}

Figures 1.1(b,c) show averaged histograms of the times at which spikes were recorded in LC neurons, in data accumulated from many stimulusresponse trials. The phasic histogram displays a post-stimulus window in which the probability of a given LC neuron firing in an arbitrary trial is relatively high; this represents increased activity following stimulus. After this burst the histogram exhibits a 'refractory' period in which there is a lower firing probability, followed by a gradual return to equilibrium firing probability. We now indicate a mechanism by which this pattern can emerge from averages over many trials of oscillator dynamics with the appropriate initial conditions.

As noted in the Introduction, the LC response in the phasic mode is stronger than in the tonic mode. This effect can be shown to arise from the fact that phasic-mode LC neurons tend to have lower firing frequencies (Brown, Moehlis, Holmes, Aston-Jones, and Clayton [2002]). Here we only study phase-difference models based on equation (4.1) for the phasicmode LC response. We assume that external stimuli act (via other neural groups) by increasing the input current to each LC neuron identically via a function $I(t)$, contributing a term $z(\theta) I(t)$ to the phase velocities. Approximating the phasic mode as perfectly synchronized so that the average 
$\frac{\alpha}{N} \sum_{j \neq i}^{n} f\left(\theta_{j}-\theta_{i}\right)$ may be replaced by the constant $f(0)$, equation (4.1) becomes

$$
d \theta=[\omega+\alpha f(0)+z(\theta) I(t)] d t+\sigma d W_{t} \triangleq v(\theta, t) d t+\sigma d W_{t} .
$$

Initial conditions $\theta(0)=\theta_{0}$ are drawn from the appropriate distribution (see below).

The probability density of oscillator phases for this stochastic ODE (4.2) evolves according to the forward Kolmogorov equation

$$
\frac{\partial \rho(\theta, t)}{\partial t}=-\frac{\partial}{\partial \theta}[v(\theta, t) \rho(\theta, t)]+\frac{\sigma^{2}}{2} \frac{\partial^{2} \rho(\theta, t)}{\partial \theta^{2}},
$$

with $\rho(\theta, t=0)=\delta\left(\theta-\theta_{0}\right)$. Due to the linearity of (4.3), histograms to be compared with Figure 1.1 may be produced from (4.3) with initial density representing an average over many trials, in which case $\rho(\theta, t) d \theta$ represents the probability that a given neuron observed in an arbitrary trial has phase in the interval $[\theta, \theta+d \theta)$ at time $t$. This 'density' approach to modeling experimental firing time histograms is also employed in Ritt and Kopell [2002]. The experimentally relevant initial condition $\rho_{0}$ may be found by reasoning as follows: if $v$ lacks explicit $t$-dependence (i.e. $I(t)=0$ ), then the probability that an oscillator obeying (4.2) is in $[\theta, \theta+d \theta]$ should scale with $1 / T(\theta)$, where $T(\theta)$ is the time spent in this interval during one cycle. To lowest order in $d \theta$ and neglecting noise, this implies $\rho(\theta, t=0)=$ $C / v(\theta)$ for some constant $C$; normalization gives $\rho_{0}$ itself, which is simply $1 / 2 \pi$ in this case. Histograms of firing times may be extracted from the solutions of (4.3) by noting that the firing probability (for an arbitrarily chosen neuron) at time $t$ is proportional to the threshold probability flux $F L(t) \triangleq v\left(\theta_{s}, t\right) \rho\left(\theta_{s}, t\right)$.

We can develop explicit solutions to (4.3) for certain classes of stimuli and PRCs in the absence of noise for comparison with experimental data; these analyses will appear in a future paper (Brown, Moehlis, Holmes, Aston-Jones, and Clayton [2002]). Figure 4.4 illustrates preliminary results relevant to the data of Figure 1.1(b,c) via numerical solutions of (4.3) and direct simulations of (4.1) (with identical stimuli), which show that $F L(t)$, derived from (4.3), provides a reasonable approximation for post-stimulus firing probabilities. We note that $F L(t)$ displays the characteristic peak and refractory period of Figures 1.1(b,c), but the subsequent return to equilibrium is slow and exhibits prolonged 'ringing' not seen in the experimental data. The ringing is due to a 'resonance' between the stimulus duration and the oscillator frequency; it is diminished or disappears entirely, giving histograms more similar to Figures 1.1(b,c), when data is averaged over a distribution of frequencies (Brown, Moehlis, Holmes, Aston-Jones, and Clayton [2002]). 

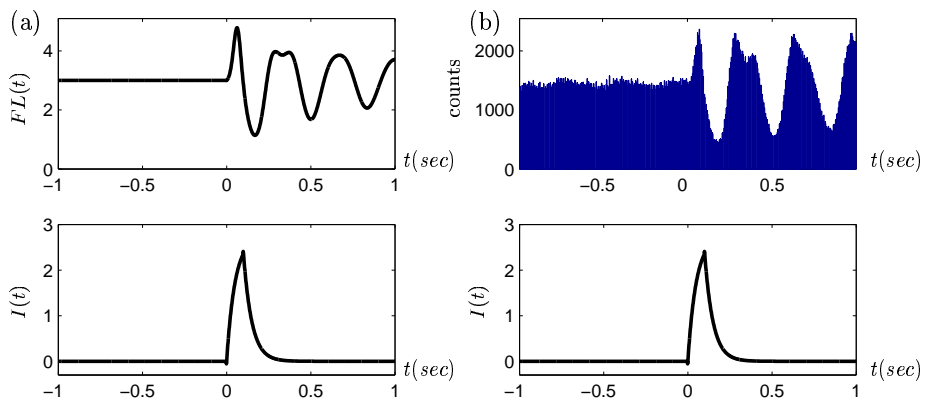

FiguRE 4.4. LC firing rates (proportional to the probability that a neuron sampled from the LC will fire at a particular time), computed using equation (4.3). Upper plot: (a) $F L(t)$, (b) firing time histogram for the corresponding finite-trial simulation, see text. Parameters: $N=24, \kappa=0, \alpha=0.032, \sigma=0.8$ (as in Figure 4.2(b)). Lower plot (a,b): $I(t)$ in $\mu A / \mathrm{cm}^{2}$.

\section{Conclusions}

The dynamics of a finite set of identically (mean field) coupled oscillators were analyzed in general cases of phase-difference coupling and in specific cases of combined phase-difference and phase-dependent 'product' coupling. The existence of symmetric equilibria and fixed tori for $S_{N} \times T^{1}$ equivariant systems of coupled oscillators was demonstrated, as was the dependence of their stability and degeneracy on the Fourier content of the coupling functions. In particular, while single harmonic sine and cosine functions are degenerate in that they give rise to steady states with multiple zero eigenvalues, the inclusion of higher harmonics generically produces equilibria with only a single (necessarily) zero eigenvalue in the relative phase direction. Illustrative examples of such functions were derived from Hodgkin-Huxley neural models, and stability and domains of attraction of synchronized periodic orbits were investigated in detail.

This analysis of idealised oscillator networks guided the numerical simulations of Section 4, which demonstrate that networks of rotators can reproduce phenomena observed in firing patterns of the brain organ locus coeruleus. Specifically, the model suggests three mechanisms potentially responsible for broadening of the phasic cross correlogram (Figure 1.1(a)): multiple equilibria with distinct phase differences, random external effects, and randomly distributed natural frequencies (Figures 4.2(b-d)). The former is perhaps least likely, since it tends to give multi-peaked correlograms. In addition, relatively strong synaptic (phase product) coupling appears necessary to reproduce the depressed firing rates of the phasic state: averaged (phase-difference) synaptic coupling actually produced the opposite effect, and electrotonic coupling does not change in-phase firing rates. Fi- 
nally, we note that these results depend subtly on synaptic delays and time constants, and thus that details of the underlying cell membrane and channel dynamics must enter the phase oscillator description. In fact in ongoing work (Brown, Moehlis, Holmes, Aston-Jones, and Clayton [2002]), we incorporate an additional slow calcium-dependent potassium current, leading to coupling functions differing from those of Figure 4.1, and, in some cases, remarkably close to the degenerate pure sinusoids.

A general point of contact between the present study and neuroscience lies in the relationship between explicitly modeling individual neurons and their couplings, and averaging the behavior of a (sub-)population into a single connectionist-type 'unit' (e.g. Rumelhart and McClelland [1986]). Domain of attraction and probability density results for synchronized states may inform conditions under which such approximations are justifiable. In the present paper, a simple probabilistic evolution (equation (4.3)) for coherent phase states produces acceptable results (Figure 4.4). Extension of this result to a true population average for systems with distributed frequencies and non-uniform couplings would bring it closer to the work of Sirovich, Knight, and Omurtag [2000], and help unify detailed (HodgkinHuxley type) neural models, simpler integrate-and-fire and phase models, and connectionist networks.

Acknowledgments: This work was partially supported by DoE: DEFG02-95ER25238 and NIMH: MH62196 (Cognitive and Neural Mechanisms of Conflict and Control, Silvio M. Conte Center). Eric Brown was supported by a National Science Foundation Graduate Fellowship and a Burroughs-Wellcome Training Grant in Biological Dynamics: 1001782, and Jeff Moehlis by a National Science Foundation Postdoctoral Research Fellowship. We thank Jason Ritt for assistance with incorporating the stimulus into the density approach as well as other helpful comments, Georgi Medvedev for useful suggestions and discussions, and Martin Golubitsky for an insightful and stimulating communication early in this project. Weinan E provided motivation for studying and insights about symmetric solutions in the $N \rightarrow \infty$ limit.

\section{References}

Arnold, V. [1973], Ordinary Differential Equations. MIT Press, Boston.

Ashwin, P. and J. Swift [1992], The dynamics of $N$ weakly coupled identical oscillators, J. Nonlin. Sci. 2, 69-108.

Aston-Jones, G., J. Rajkowski, and T. Alexinsky [1994], Locus coeruleus neurons in the monkey are selectively activated by attended stimuli in a vigilance task., J. Neurosci. 14, 4467-4480. 
Bressloff, P. and S. Coombes [1998], Spike train dynamics underlying pattern formation in integrate-and-fire oscillator networks, Phys. Rev. Lett. 81, 23842387.

Brown, E., J. Moehlis, P. Holmes, G. Aston-Jones, and E. Clayton [2002], The influence of spike rate on response in locus coeruleus, Unpublished manuscript, Program in Applied and Computational Mathematics, Princeton University, 2002.

Chow, C. and N. Kopell [2000], Dynamics of spiking neurons with electrotonic coupling, Neural Comp. 12, 1643-1678.

Crawford, J. [1995], Scaling and singularities in the entrainment of globally coupled oscillators, Phys. Rev. Lett. 74, 4341-4344.

Crawford, J. and K. Davies [1999], Synchronization of globally coupled phase oscillators: singularities and scaling for general couplings, Physica D 125 (12), 1-46.

Daido, H. [1994], Generic scaling at the onset of macroscopic mutual entrainment in limit cycles with uniform all-to-all coupling, Phys. Rev. Lett. 73(5), 760763.

E, W. [2001], personal communication, 2001.

Ermentrout, B. [1996], Type I membranes, phase resetting curves, and synchrony, Neural Comp. 8, 979-1001.

Ermentrout, G. and N. Kopell [1990], Oscillator death in systems of coupled neural oscillators, SIAM J. on Appl. Math. 50, 125-146.

Fenichel, N. [1971], Persistence and smoothness of invariant manifolds for flows, Ind. Univ. Math. J. 21, 193-225.

Freidlin, M. and A. Wentzell [1998], Random perturbations of dynamical systems. Springer, New York.

Gerstner, W., L. van Hemmen, and J. Cowan [1996], What matters in neuronal locking?, Neural Comp. 8, 1653-1676.

Golomb, D., D. Hansel, B. Shraiman, and H. Sompolinsky [1992], Clustering in globally coupled phase oscillators, Phys. Rev. A 45(6), 3516-3530.

Golubitsky, M., I. Stewart, and D. Schaeffer [1988], Singularities and Groups in Bifurcation Theory, Vol. 2. Springer, New York.

Grant, S., G. Aston-Jones, and D. Redmond [1988], Responses of primate locus coeruleus neurons to simple and complex sensory stimuli., Brain Res. Bull. 21 (3), 401-410.

Guckenheimer, J. and P. Holmes [1983], Nonlinear Oscillations, Dynamical Systems and Bifurcations of Vector Fields. Springer-Verlag, New York.

Hansel, D., G. Mato, and C. Meunier [1995], Synchrony in excitatory neural networks, Neural Comp. 7, 307-337.

Hodgkin, A. and A. Huxley [1952], A quantitative description of membrane current and its application to conduction and excitation in nerve, J. Physiol. 117, 500-544.

Honeycutt, R. [1992], Stochastic Runge-Kutta Algorithms I. White Noise, Phys. Rev. A 45, 600-603. 
Hoppensteadt, F. and E. Izhikevich [1997], Weakly Connected Neural Networks. Springer-Verlag, New York.

Izhikevich, E. [2000], Phase equations for relaxation oscillators, SIAM J. on Appl. Math. 60, 1789-1804.

Johnston, D. and S. Wu [1997], Foundations of Cellular Neurophysiology. MIT Press, Cambridge, MA.

Keener, J. and J. Sneyd [1998], Mathematical Physiology. Springer, New York.

Kim, S. and S. Lee [2000], Phase dynamics in the biological neural networks, Physica D 288, 380-396.

Kopell, N. and G. Ermentrout [1990], Phase transitions and other phenomena in chains of coupled oscillators, SIAM J. on Appl. Math. 50, 1014-1052.

Kopell, N. and G. Ermentrout [1994], Inhibition-produced patterning in chains of coupled nonlinear oscillators, SIAM J. Appl. Math. 54, 478-507.

Kopell, N., G. Ermentrout, and T. Williams [1991], On chains of osciallators forced at one end, SIAM J. on Appl. Math. 51, 1397-1417.

Kuramoto, Y. [1984], Chemical Oscillations, Waves, and Turbulence. Springer, Berlin.

Kuramoto, Y. [1997], Phase- and center-manifold reductions for large populations of coupled oscillators with application to non-locally coupled systems, Int. J. Bif. Chaos 7, 789-805.

Murray, J. [2001], Mathematical Biology, 3rd. Ed. Springer, New York.

Nichols, S. and K. Wiesenfeld [1992], Ubiquitous neutral stability of splay states, Phys. Rev. A 45(12), 8430-8435.

Okuda, K. [1993], Variety and generality of clustering in globally coupled oscillators, Physica D 63, 424-436.

Omurtag, A., E. Kaplan, B. Knight, and L. Sirovich [2000a], A population approach to cortical dynamics with an application to orientation tuning, Network 11, 247-260.

Omurtag, A., B. Knight, and L. Sirovich [2000b], On the simulation of large populations of neurons, J. Comp. Neurosci. 8, 51-63.

Ritt, J. and N. Kopell [2002], In preparation, 2002.

Rumelhart, D. and J. McClelland [1986], Parallel Distributed Processing: Explorations in the Microstructure of Cognition. MIT Press, Cambridge, MA.

Servan-Schreiber, D., H. Printz, and J. Cohen [1990], A network model of catecholamine effects: Gain, signal-to-noise ratio, and behavior, Science 249, 892895.

Sirovich, L., B. Knight, and A. Omurtag [2000], Dynamics of neuronal populations: The equilibrium solution, SIAM J. on Appl. Math. 60, 2009-2028.

Strogatz, S. [2000], From Kuramoto to Crawford: Exploring the onset of synchronization in populations of coupled oscillators, Physica D 143, 1-20.

Taylor, D. and P. Holmes [1998], Simple models for excitable and oscillatory neural networks, J. Math. Biol. 37, 419-446.

Tsang, K., R. Mirollo, and S. Strogatz [1991], Dynamics of a globally coupled oscillator array, Physica D 48, 102-112. 
Usher, M., J. Cohen, D. Servan-Schreiber, J. Rajkowsky, and G. Aston-Jones [1999], The role of locus coeruleus in the regulation of cognitive performance, Science 283, 549-554.

van Vreeswijk, C., L. Abbot, and B. Ermentrout [1994], When inhibition not excitation synchronizes neural firing, J. Comp. Neurosci. 1, 313-321.

Watanabe, S. and S. Strogatz [1994], Constants of the motion for superconducting Josephson arrays, Physica D 74, 195-253.

Watanabe, S. and J. Swift [1997], Stability of periodic solutions in series arrays of Josephson Junctions with internal capacitance, J. Nonlin. Sci. 7, 503-536.

Weisenfeld, K., P. Colet, and S. Strogatz [1998], Frequency locking in Josephson arrays: Connection with the Kuramoto model, Phys. Rev. E 57 (2), 15631569.

Wiggins, S. [1994], Normally Hyperbolic Invariant Manifolds in Dynamical Systems. Springer, New York.

Williams, J., R. North, A. Shefner, S. Nishi, and T. Egan [1984], Membrane properties of rat locus coeruleus neurons, Neuroscience 13, 137-156.

Zhu, W. [1988], Stochastic averaging methods in random vibration, Appl. Mech. Rev. 41, 189-199. 